\title{
Superinfection of Lysogenic Strains of Salmonella typhimurium Q1: Prophage Substitution and Double Lysogenization
}

\author{
BY J. S. K. BOYD AND D. E. BIDWELL \\ The Wellcome Laboratories of Tropical Medicine, London, N.W. 1
}

(Received 23 March 1962)

\begin{abstract}
SUMMARY
Strains of Salmonella typhimurium Q1, lysogenized with type A phages, were superinfected with the heterologous free phages of the same group. This produced lysis (productive or vegetative development) and prophage change (either prophage substitution or double lysogenization) in a constant pattern. Prophage change was frequently detected when lysis was absent. Certain of the phages were aggressive, producing active lysis and prophage change in many of the heterologous lysogenic strains, others were intermediate and some were non-aggressive. In general, aggressive phages in prophage form conferred a good degree of immunity on the host bacterium, while non-aggressive phages did not: but to this rule there were exceptions. In most cases, immunity to lysis and immunity to prophage change ran an approximately parallel course, but again there were exceptions. Some strains, with certain superinfections, were immune to lysis but not to prophage change, while others showed greater resistance to prophage change than to lysis. The reaction to superinfection split the series into two groups. Superinfection of a lysogenic organism of either group with phage of the same group produced-if there was any prophage change-prophage substitution: superinfection of a lysogenic organism of one group with phage of the other group produced double lysogenization. Each group of phages had therefore its own site of attachment to the bacterial chromosome. Immunity appeared to be due neither to defective adsorption nor to steric interference, but to repressors with specific characters which varied from strain to strain.
\end{abstract}

\section{INTRODUCTION}

Lysogenic bacteria are generally (but not invariably) immune to the temperate phage which they produce, and in some cases, but in a more limited way, to related temperate phages of the same group. The cross-immunity test, used to differentiate and identify twelve of the temperate Type A phages found in Salmonella typhimurium, is based on the varying degrees of resistance found in bacteria lysogenized by these phages (Boyd \& Bidwell, 1957). Absence of resistance is shown by plaque formation resulting from the lytic action of the superinfecting phage. In addition to producing lysis, the superinfecting phage may bring about changes in the prophage content of some of the bacteria which escape lysis (Boyd, 1956). Briefly, the superinfecting phage may either evict and replace the prophage of the nonimmune lysogenic bacteria-'prophage substitution'; or it may itself become prophage without disturbing the resident prophage, thus causing double lysogeniza- 
tion in the bacterium. In certain cases 'cure' has been noted, where the superinfecting phage has evicted the resident prophage, but has failed to establish itself as a replacement. These phenomena have now been investigated in some detail, and in this paper an account is given of the more important findings.

\section{METHODS}

The cultures and bacteriophages used in these experiments, and also the culture media and fundamental techniques, have been fully described in previous papers (Boyd, 1950; Boyd \& Bidwell, 1957, 1959, 1961). The terms adopted to designate cultures and phages are those in common use. Salmonella typhimurium $\mathbf{Q 1}$ is referred to as Q1 : phage A $1 a$ is simply A $1 a$. Q1 carrying prophage A $1 a$ (i.e. lysogenic) is $\mathrm{Q} \mathbf{1}(\mathbf{A} 1 a)$. The expression 'prophage change' is used to indicate either prophage substitution or double lysogenization. In quoting phage: bacteria ratios the phage is always shown first: thus a 1:10 ratio indicates 1 phage particle to 10 bacteria.

The first method used to establish the pattern of prophage change was based on the cross-immunity test already described (Boyd \& Bidwell, 1957). A loopful of the superinfecting phage - at least $10^{10}$ particles $/ \mathrm{ml}$.-was placed on a lawn of the lysogenic bacteria, and the plate incubated overnight at $37^{\circ}$. Whether a patch formed or not (a reaction indicating the occurrence or otherwise of lytic action of the superinfecting phage on the lysogenic bacteria), a small quantity of the material from the centre of the spot where the phage had been placed on the lawn was picked up with a sterile platinum needle and rubbed into $1 \mathrm{ml}$. of sterile broth in a test tube. A drop of this suspension was then spread on nutrient agar and incubated overnight. Accurately gauged quantities produced a plate peppered with discrete colonies. When the superinfecting phage had a lytic action on the lysogenic bacterium, some of these colonies were phage-contaminated, and presented the characters described in an earlier paper (Boyd, 1951): others were normal in appearance. Twenty colonies of normal appearance were selected, cultured in broth, and replated to confirm the absence of surface phage contamination. Those which showed evidence of contamination were discarded. Thereafter the colonies were subjected to various tests to determine their prophage content. As there were in all 132 different combinations of phage and lysogenic organisms, it is impracticable to give details of the exact steps in each identification. In most cases a modified cross-immunity test provided a simple and reliable means of determining if any prophage change had taken place. In a few it was found helpful to propagate the phage on Salmonella typhimurium strain 1404, which supports the growth of the A1 group, A3, and A4, but not the A2 group. This selective propagation was of particular value in some of the double lysogenizations, where the separation of the two phages occasionally presented considerable difficulty. Where necessary the identity of a phage was confirmed by a full-scale cross-immunity test. Control tests were made which showed that external phage contamination was not a source of error. Where the superinfecting phage lysed and so 'marked' the colony, this danger did not arise, as it was an easy matter to select uninfected colonies from a plate. In the absence of such lytic action, there was no marking of contaminated colonies. However, as the absence of lysis showed that there was no active propaga- 
tion of the superinfecting phage, the chance of any residual free phage remaining in cultures made from individual colonies, each the product of a single organism, was negligible, especially when this process was twice repeated. Selected doubly lysogenized strains, repeatedly subcultured, maintained their characters indefinitely. Further, as will be seen later, the fact that either prophage substitution or double lysogenization occurred in a definite and predictable pattern afforded convincing confirmation of the reliability of this finding.

Table 1. Method 1: prophage change

The denominator shows the number of colonies examined, the numerator the number in which prophage change-either substitution or double lysogenization-occurred.

Salmonella typhimurium Q1 carrying prophage

\begin{tabular}{|c|c|c|c|c|c|c|c|c|c|c|c|c|}
\hline $\begin{array}{l}\text { Phage type } \\
\text { Ala }\end{array}$ & A $1 a$ & $\begin{array}{c}\mathrm{A} 1 b \\
13 / 20\end{array}$ & $\begin{array}{c}\text { A } 1 c \\
0 / 20\end{array}$ & $\begin{array}{c}\text { A1d } \\
10 / 20\end{array}$ & $\begin{array}{r}\mathbf{A 2 a} \\
\mathbf{7} / \mathbf{2 0}\end{array}$ & $\begin{array}{c}A 2 b \\
0 / 20\end{array}$ & $\begin{array}{c}\mathrm{A} 2 f \\
0 / 20\end{array}$ & $\begin{array}{l}\text { A3 } \\
0 / 20\end{array}$ & $\begin{array}{l}\text { A4 } \\
0 / 20\end{array}$ & $\begin{array}{l}\mathrm{A} 2 c \\
1 / 20\end{array}$ & $\begin{array}{c}\mathrm{A} 2 d \\
4 / 20\end{array}$ & $\begin{array}{l}\mathrm{A} 2 e \\
3 / 40\end{array}$ \\
\hline Alb & $17 / 20$ & & $1 / 20$ & $10 / 20$ & $8 / 50$ & $29 / 109$ & $17 / 49$ & $4 / 40$ & $0 / 20$ & $7 / 24$ & $2 / 20$ & $4 / 20$ \\
\hline $\mathrm{A} 1 c$ & $15 / 19$ & $16 / 20$ & . & $11 / 20$ & $6 / 20$ & $10 / 18$ & $14 / 20$ & $4 / 40$ & 0 & 20 & 20 & $16 / 20$ \\
\hline Ald & $12 / 20$ & $17 / 20$ & $0 / 20$ & · & $0 / 20$ & $0 / 20$ & $10 / 40$ & $10 / 10$ & o/ & & & $20 / 20$ \\
\hline A $2 a$ & $2 / 18$ & $15 / 20$ & $0 / 20$ & $0 / 20$ & . & $9 / 20$ & $13 / 20$ & $8 / 40$ & & 20 & 20 & $16 / 20$ \\
\hline $\mathrm{A2} b$ & $13 / 19$ & $43 / 57$ & $1 / 44$ & $14 / 20$ & $11 / 20$ & · & $14 / 20$ & $7 / 30$ & $2 / 60$ & $\mathbf{2 0}$ & 20 & $5 / 18$ \\
\hline A2 $f$ & $0 / 60$ & $5 / 20$ & $0 / 20$ & $0 / 20$ & $1 / 20$ & $0 / \mathbf{2 0}$ & . & $0 / 20$ & $0 / 20$ & & $2 / 40$ & $3 / 40$ \\
\hline A3 & $0 / 40$ & $5 / 20$ & $0 / 20$ & $2 / 20$ & $4 / 20$ & $0 / 20$ & $0 / 20$ & . & $0 / 40$ & $5 / 20$ & $2 / 20$ & $2 / 20$ \\
\hline A4 & $31 / 40$ & $10 / 20$ & $0 / 20$ & $0 / 20$ & $\mathbf{0} / \mathbf{2 0}$ & $1 / 20$ & $13 / 39$ & & . & $5 / 20$ & $2 / 20$ & $10 / 20$ \\
\hline A $2 c$ & $28 / 44$ & $44 / 44$ & $0 / 60$ & $1 / 20$ & $4 / 20$ & $12 / 36$ & $15 / 40$ & & $0 / 20$ & . & $4 / 20$ & $10 / 20$ \\
\hline A2d & $13 / 20$ & $3 / 20$ & & $\mathbf{3} / \mathbf{1 9}$ & & $7 / 20$ & & & $\mathbf{0} / \mathbf{2 0}$ & $1 / 20$ & . & $11 / 2$ \\
\hline A2e & $15 / 20$ & $10 / 20$ & $16 / 20$ & $24 / 40$ & $13 / 20$ & $3 / 24$ & $13 / 40$ & $35 / 40$ & $23 / 30$ & $4 / 20$ & $15 / 20$ & 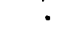 \\
\hline
\end{tabular}

In Table 1 figures are given with the number of colonies showing prophage change as the numerator and the number examined as the denominator. These give only a rough indication of the degree of reaction, not an exact quantitative measurement. Nevertheless, when repeated tests were made the results as a rule showed no gross variation, and the general picture which the figures present may be regarded as of some value, particularly at the lower limits. This simple technique will be referred to as method 1. A more precise quantitative estimate of the degree of prophage substitution or double lysogenization and of the associated productive infection (vegetative development or bursts) was obtained by means of two different techniques.

In certain cases (e.g. Q1 (A 1 $a$ ) superinfected with A1b) a modification of Levine's (1957) method was used. Salmonella typhimurium $\mathbf{T}$ gal-, kindly given to us by Dr Prell, is sensitive to Al $a$, and it was therefore possible to prepare a lysogenic strain, $\mathrm{T} \mathrm{gal}^{-}$(A $1 a$ ), which was immune to A $1 a$. Q1 is gal ${ }^{+}$, and so outgrew $\mathbf{T}$ gal ${ }^{-}$(A $1 a$ ) when plated as a dilute suspension on a lawn of this organism on galactose agar. A $1 b$ produced plaques freely on $\mathbf{T}$ gal- $^{-}(\mathbf{A} 1 a)$. Thus on a lawn of $\mathbf{T}$ gal- $^{-}(\mathrm{A} 1 a)$, bursts liberating $\mathbf{A} 1 b$ produced plaques best seen after incubation for $24 \mathrm{hr}$. at $37^{\circ}$, while colonies of $\mathrm{Q} 1(\mathrm{Al} b)$ could be distinguished by the halo which surrounded them when, after $48 \mathrm{hr}$. incubation at $37^{\circ}$, the plates were kept for a further $24 \mathrm{hr}$. at room temperature. On the other hand Q1 (A $1 a$ ) gave colonies which, by virtue of their more vigorous growth, stood out from the lawn of $\mathrm{T}_{\text {gal- }}$ (A 1 a), but had no surrounding halo. In tests by this method, cultures of the lysogenic strain in the logarithmic phase of growth, diluted to a standard concentra- 
tion ( $10^{8}$ organisms $/ \mathrm{ml}$.) were exposed to graded concentrations of the superinfecting phage and placed in the water bath at $37^{\circ}$ for $10 \mathrm{~min}$. Thereafter each culture was diluted $10^{-5} \times 1 / 5$ (using, at an intermediate stage in the process of dilution, an antiphage serum to neutralize any non-adsorbed free phage), $0.5 \mathrm{ml}$. quantities were gently flooded on to a lawn of T gal $^{-}(\mathrm{Al} a)$ on galactose agar, and plaques and colonies counted at the times indicated. A count of the viable bacteria in a control culture provided a figure from which could be calculated the percentage of bursts and of bacteria in which prophage substitution had occurred. Unfortunately this method, which will be designated method 2 , has only a limited application. $\mathbf{T}$ gal- is resistant or partly resistant to several phages of the series, and of course double lysogenizations cannot be investigated in this way.

The preliminary steps of method 3 were similar to those in method 2, but the diluted suspension was plated directly on nutrient agar, and not on a lawn of lysogenized $\mathbf{T}$ gal- $^{-}$on galactose agar. Each colony which developed was tested for its prophage content. Bursts were calculated by the 'tube' technique (Boyd \& Bidwell, 1959).

\section{RESULTS}

\section{Preliminary investigations}

Using method 1, each lysogenic strain was exposed to superinfection with the heterologous temperate phages of the series. It was at first assumed that the pattern of reactions would be similar to that of the cross-immunity test, and that prophage substitution or double infection would occur in parallel with the lysis which produced the patches or plaques in the cross-immunity test. This assumption

Table 2. Superinfection with A1 $b$ : prophage change in the lysogenic bacteria of the series

The figures give the percentage of bacteria in which prophage change occurred, estimated by method 3. Q1, exposed to $A 1 b$ in a $1: 1$ ratio, showed approximately $45 \%$ lysogenization. A1 $a$ therefore afforded no protection: A1c, A4, A2d and A2e gave $100 \%$ immunity at this ratio: the others fell between these extremes.

Exposed to $A 1 b$ in $1: 1$ ratio

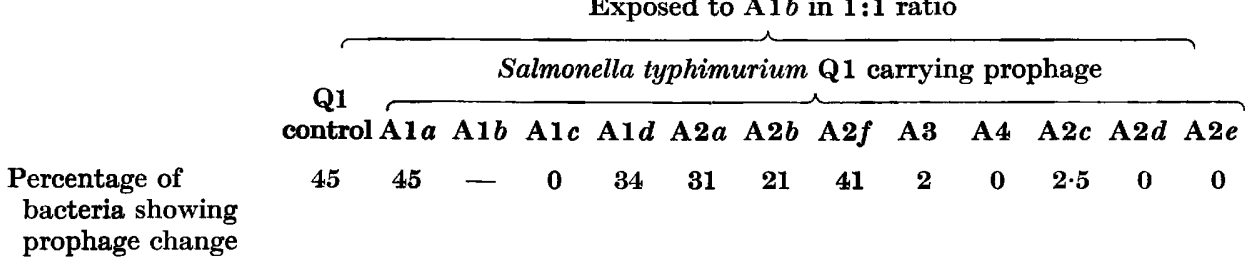

proved to be wrong, for it was found that in some cases, where there was no detectable lysis, superinfection gave rise to prophage change. The results are shown graphically in Fig. 1, while in Table 1 figures are given which, subject to the reservations already made, give some indication of the degree of prophage change.

A more accurate estimation of the varying percentages of prophage change in the lysogenic strains superinfected with $\mathrm{Al} b$ at a $1: 1$ ratio is given in Table 2, and reference will be made in the text to results given by method 3 in other cases. A remarkable feature of these findings was the division of the twelve lysogenic strains into two groups differentiated by the occurrence of prophage substitution 
or double lysogenization. This phenomenon will be fully discussed at a later stage. Apart from this one definite pattern, the picture is very complex. In an attempt to throw some light on its many puzzling aspects, numerous investigations were made, from which the following examples have been selected to illustrate the different types of reaction which have been encountered.

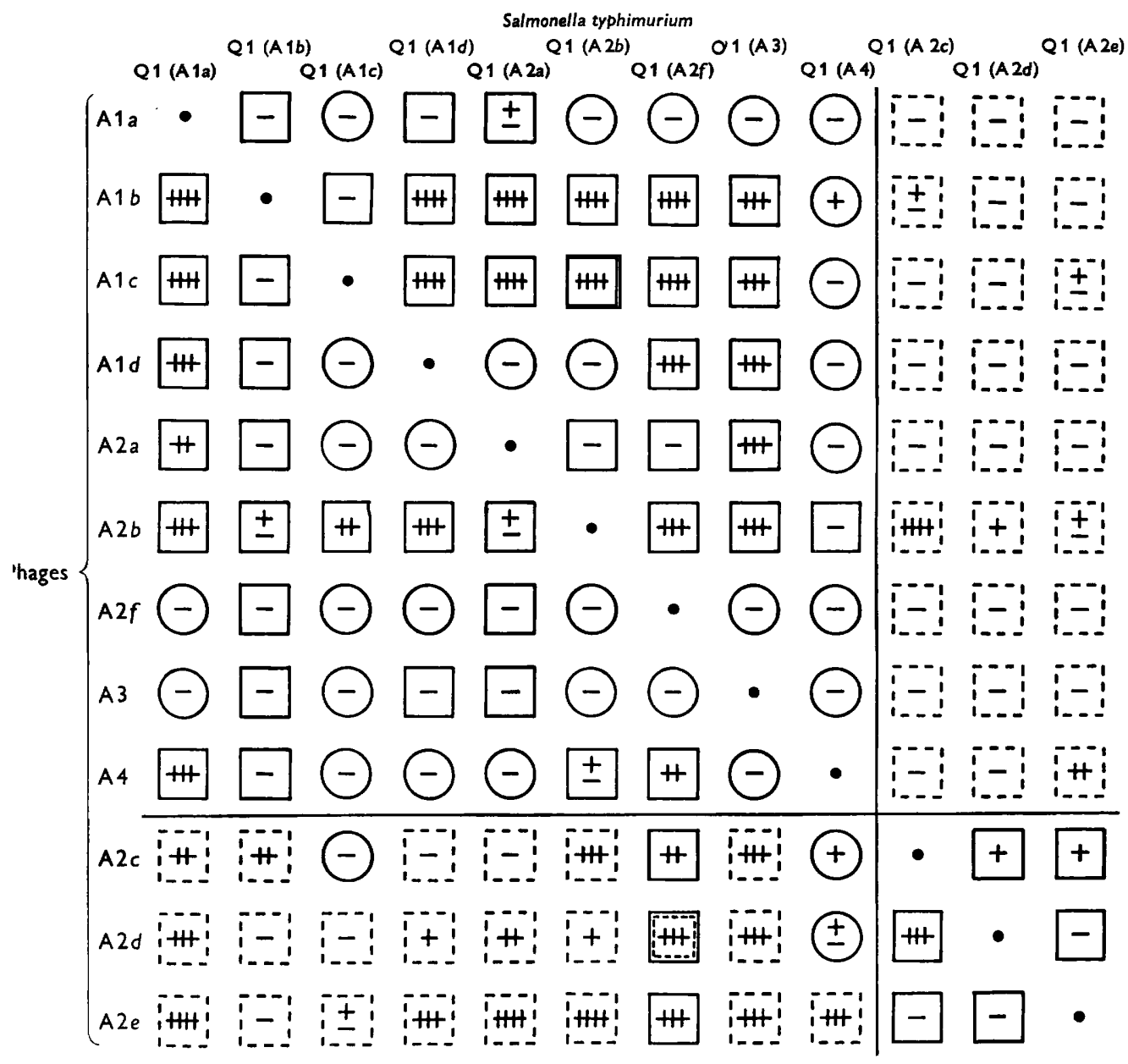

Fig. 1. Graphic record of lysis and lysogenesis resulting from superinfection, as shown by method 1. $\square$, Prophage substitution; $\square$, double lysogenization; $O$, no prophage change; $\bullet$, not tested; ++++ to - , degree of lysis at critical test concentration of phage (lowest concentration giving confluent lysis in control). Note: A2d against Q1 (A2 f ) 7/60 double lysogenization, 25/60 prophage substitution; A2 $e$ against Q1 (A1 $d$ ), one colony found showing prophage substitution.

\section{Prophage substitution}

$\mathrm{A} 1 a \rightleftharpoons \mathrm{A} 1 b$. Prophage substitution is well illustrated by the action of A1b on Q1 (A1 $a$ ). In terms of the cross-immunity test, this falls into the category of one-way reactions, as $A 1 a$ does not produce visible lysis in $Q 1(A 1 b)$, while the lytic action of $\mathrm{A} 1 b$ on $\mathrm{Q} 1(\mathrm{~A} 1 a)$ is well marked. Thus the plating efficiency of $\mathrm{A} 1 b$ 
on Q1 (A $1 a$ ) was virtually identical with its plating efficiency on non-lysogenic Q1, the plaque counts of the batch of $A 1 b$ used in these tests being $1 \cdot 76 \times 10^{10} / \mathrm{ml}$. on Q1, and $1.7 \times 10^{10} / \mathrm{ml}$. on Q1 (A $\left.1 a\right)$. The plaques formed on the two strains appeared identical. A1 $b$ was equally well adsorbed on Q1 and Q1 (A $1 a$ ) (Fig. 2). The opacity curves of growing cultures of Q1 and Q1 (A $1 a$ ) exposed to different concentrations of $\mathrm{A} 1 b$ were alike, indicating corresponding degrees of vegetative development and

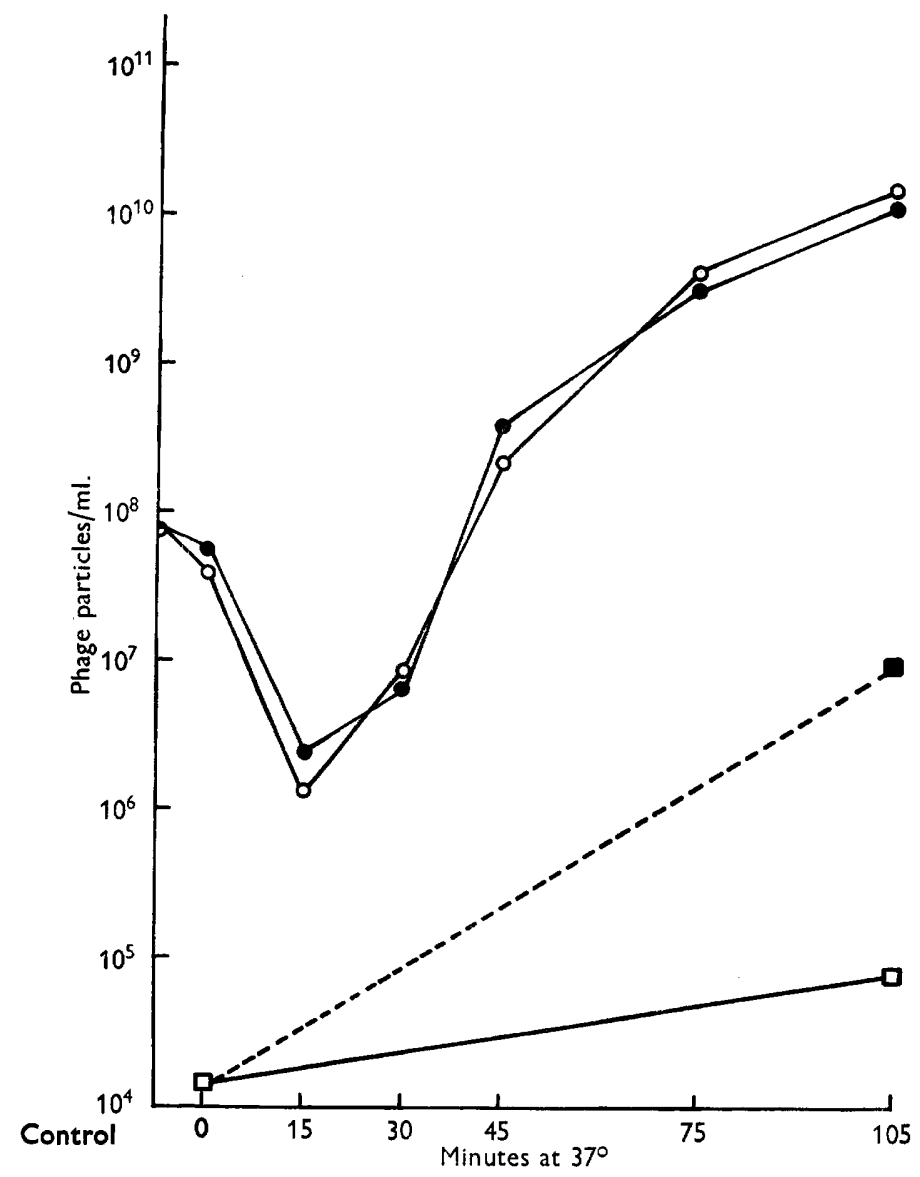

Fig. 2. Adsorption of A1 $b$ on Q1 and Q1 (A1 $a$ ). Phage:bacteria ratio, in this and all other experiments unless specifically stated, was approximately $1: 1$. Incubation was in a water-bath at $37^{\circ}$. The pre-zero fall in phage titre was due to adsorption during the process of mixing before a specimen could be taken. Samples were removed at the intervals shown, immediately diluted in decimal steps to $10^{-6}$ to prevent further adsorption, and heated to $\mathbf{7 0}^{\circ}$ for $30 \mathrm{~min}$. to kill the bacteria. Thereafter counted by standard method. All counts are plaque counts. Adsorption after $15 \mathrm{~min}$. was obscured by phage production. There was no significant difference in the curves. There was some induction of Q1 (A1 $a$ ) by A1 $b$. O, A $1 b$ on Q1; , A $1 b$ on Q1 (A1 $a)$;, A $1 a$ from induction of Q1 (A 1 $a) ; \square, A 1 a$ in control culture of Q1 (A $1 a)$.

presumably of lysogenization (Fig. 3). The adapted Levine technique was used to determine in some detail the reactions of Q1 (A 1 $a$ ) superinfected with A1 $b$. The results are shown in comparison with results given by $\mathrm{Q} 1$ exposed to $\mathrm{A} 1 b$ (Table 3 ). 
The similarity is striking, and indicates that prophage Al $a$ confers no immunity against A1b. To control the results given by the Levine technique, cultures of Q1 (A $1 a$ ), which had been exposed to A $1 b$ in a $1: 1$ ratio, were prepared in the same way as in the foregoing experiment, and cultured on plain nutrient agar. All colonies which developed on incubation were examined. The proportions which proved to be Q1 (A1 $a$ ) and Q1 (A1 $b$ ) were in good agreement with the figures given by the Levine technique: in addition, $1 \%$ were found to be 'cured', i.e. did not contain either prophage Al $a$ or prophage $\mathrm{A} 1 b$.

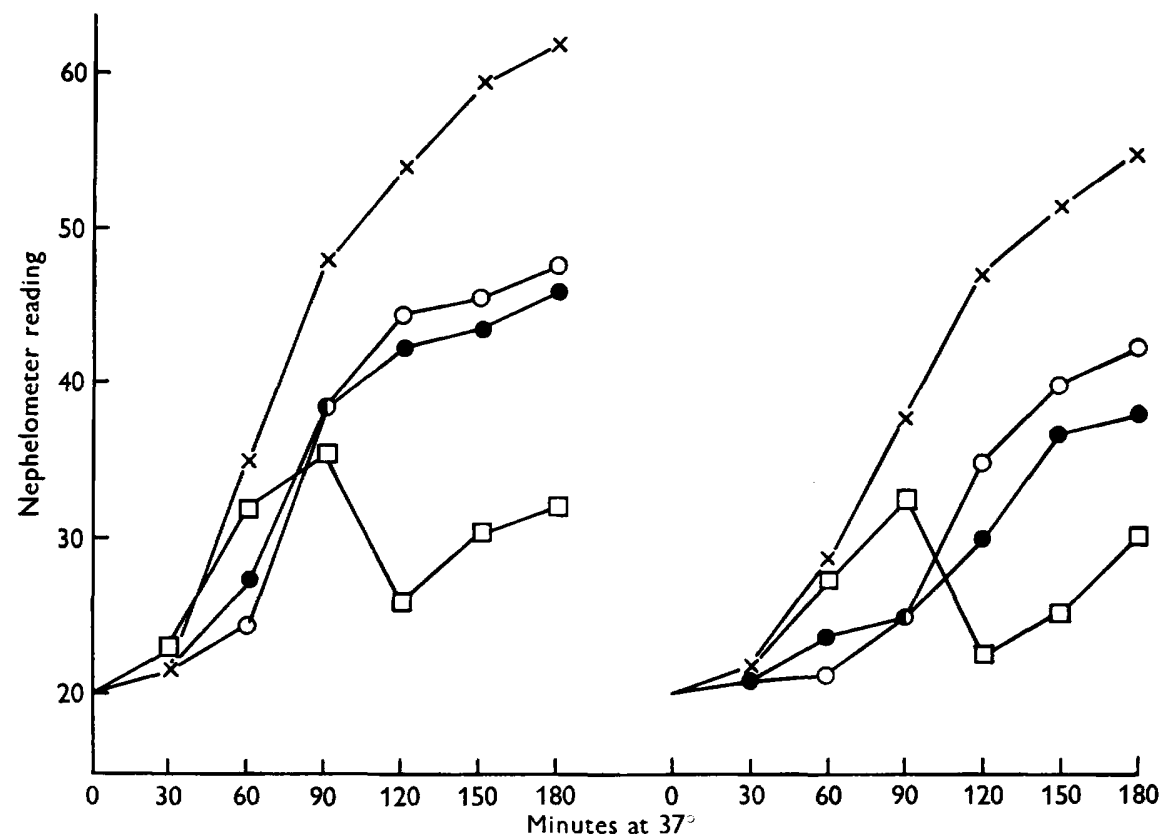

Fig. 3. Opacity curves of growing cultures of Q1 and Q1 (A $1 a$ ) exposed to A $1 b$. There was no significant difference. $x$, Control; $0,10: 1$ ratio; $, 1: 1$ ratio; $\square, 0 \cdot 1: 1$ ratio.

Table 3. Comparison of $Q 1$ infected and $Q 1$ ( $A 1$ a) superinfected with $A 1 \mathrm{~b}$

The bacteria were throughout in a concentration of $10^{8} / \mathrm{ml}$.; phage was added in quantities giving the appropriate ratio. Lysogenics and bursts were estimated by the adapted Levine technique. The figures are the percentage of bacteria in which prophage change or bursts occurred.

\begin{tabular}{|c|c|c|c|c|c|c|c|c|}
\hline $\begin{array}{c}\text { Phage: bacteria } \\
\text { ratio } \ldots\end{array}$ & & $1: 1$ & & $31: 1$ & & $1: 1$ & & $3 \cdot 1: 1$ \\
\hline $\begin{array}{l}\text { Phage Al } b \text { in- } \\
\text { fecting or super- } \\
\text { infecting }\end{array}$ & Q1 & Q1 $(\mathrm{A} \mid a)$ & Q1 & Q1 $(\operatorname{Al} a)$ & Q1 & Q1 (A1a) & Q1 & Q1 $(A 1 a)$ \\
\hline $\begin{array}{l}\text { Lysogenized with } \\
\text { A1 } b\end{array}$ & $7 \cdot 3$ & $7 \cdot 2$ & $16 \cdot 3$ & $18 \cdot 5$ & 43 & 45 & 64.5 & 70 \\
\hline Bursts & $2 \cdot 6$ & 5.5 & 10 & $9 \cdot 4$ & 24.5 & $22 \cdot 8$ & 12 & 17 \\
\hline
\end{tabular}

In contrast to this clear-cut picture, the reactions of $Q 1(A 1 b)$ superinfected with A1 $a$ were less obvious. A1 $a$ did not form plaques on Q1 (A1b) and this absence of lytic action was confirmed both by the opacity curves which showed 
no evidence of clearing at any of the phage: bacteria ratios, and by the adsorption test which showed that, although A1 $a$ was well adsorbed to Q1 (A1b), there was no subsequent increase in free phage resulting from vegetative development (Fig. 4). However, when examined by method 1, and when bacteria from the apparently negative 'patch' of A1 $a$ on Q1 (A1b) were plated, 13 out of 20 colonies selected

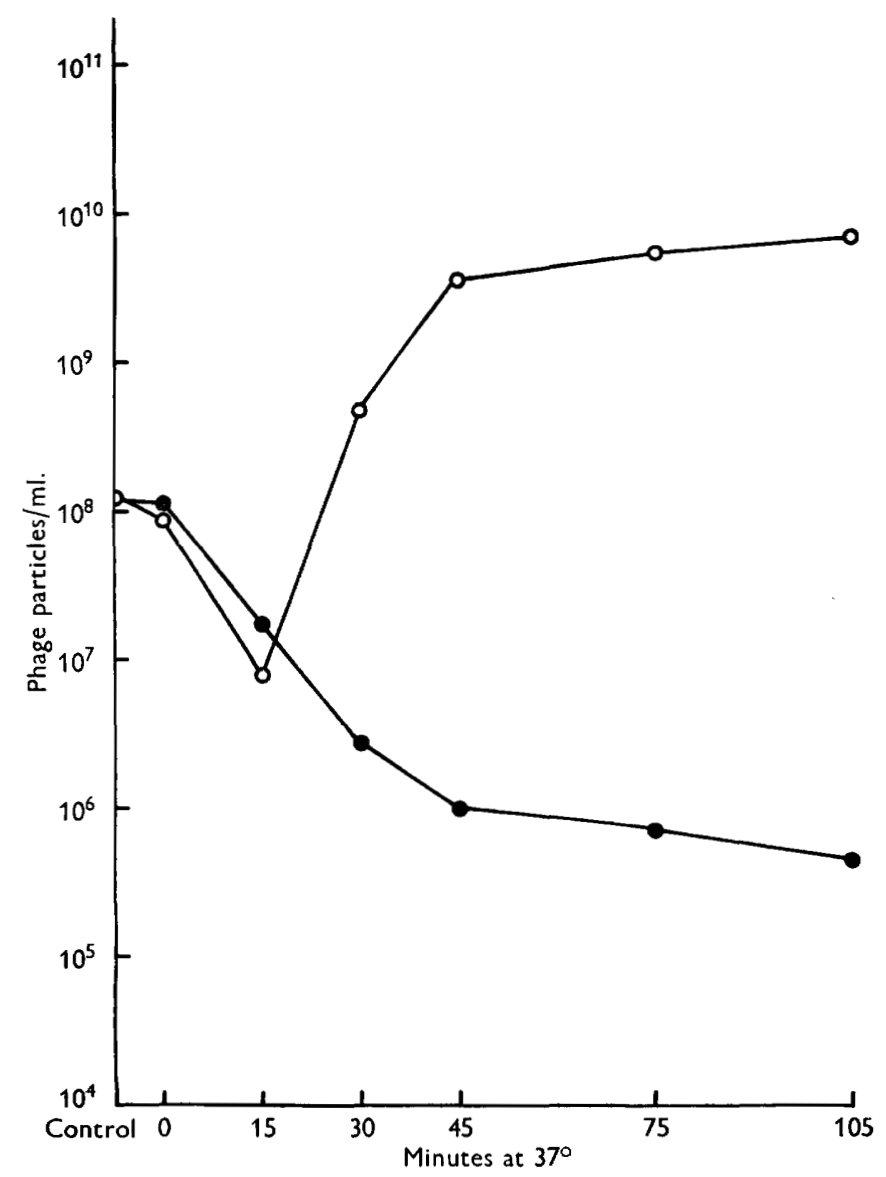

Fig. 4. Adsorption of A1 $a$ on Q1 and Q1 (A1 $b$ ). Method as deseribed for Fig. 2. On Q1, adsorption obscured after $15 \mathrm{~min}$. by phage production, $O$; on $Q 1(\mathrm{Al} b)$, adsorption without phage production, 9 .

for further tests proved to be sensitive to the action of A $1 b$, and gave the reactions of $\mathrm{Q} 1(\mathrm{Al} a)$ in a full-scale cross-immunity test, while by method 3 at a 10:1 ratio prophage substitution took place in $1.7 \%$ of the superinfected bacteria. Thus despite the absence of detectable lysis an appreciable degree of prophage substitution had occurred, the 'aggressive' Al $b$ prophage having been evicted and replaced by the apparently much less active AI $a$. No 'cures' occurred among the colonies tested.

$A 1 \mathrm{~b} \rightleftharpoons A 2 \mathrm{~b}$. These two phages have a more balanced reaction: each produces plaques-i.e. lysis - on the opposite lysogenic organism, though Al $b$ is the more active of the two. On Q1 (A $2 b$ ) the plating efficiency of $A 1 b$ was more than halved, 
the plaque count of the batch of phage used being $1 \cdot 1 \times 10^{10} / \mathrm{ml}$. on $Q 1$ and $4 \cdot 8 \times$ $10 \% / \mathrm{ml}$. on Q1 (A2b). The plaques on Q1 (A $2 b$ ) had a granular centre, and showed gross variation in size. Adsorption of $\mathrm{A} 1 b$ on $\mathrm{Q} 1(\mathrm{A2} b)$ and subsequent production of free phage showed a lag of $15 \mathrm{~min}$. when compared with similar reactions between A1 $b$ and Q1 (Fig. 5). Opacity curves of cultures of Q1 (A2b) exposed to

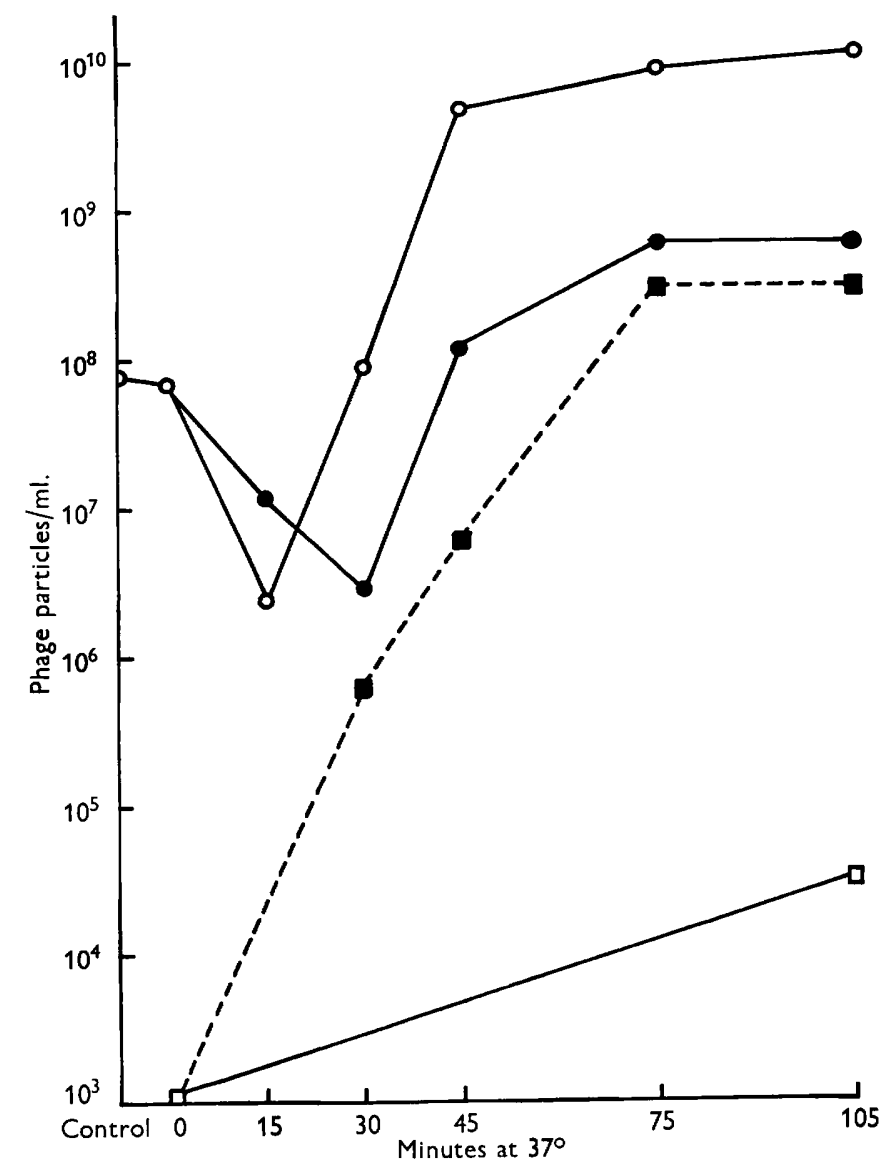

Fig. 5. Adsorption of A1b on Q1 and Q1 (A 2b). Adsorption on Q1 (A2b) slower and phage production more limited. A1 b both multiplied in and induced Q1 (A2b). $O, A 1 b$ adsorbed on Q1;, on $Q 1$ (A2b);,$A 2 b$ from induction of Q1 (A2b); $\square, A 2 b$ in control culture of Q1 (A2b).

$A 1 b$ in a ratio of $1: 10$ showed well-marked clearing after $120 \mathrm{~min}$. incubation, whereas in a control with Q1 the clearing began in 90 min.

Because of the low plating efficiency and in particular the atypical plaques, Levine's technique proved unsatisfactory, and the degree of conversion was determined by method 3, using a $1: 1$ ratio. 150 of the resulting colonies were tested for lysogenicity, and 30 proved to be Q1 (A $1 b)$, while 3 were cures, the remainder being unchanged $\mathrm{Q} 1(\mathbf{A} 2 b)$. Calculated in terms of the count of viable bacteria in control cultures, this amounted to a conversion rate from Q1 (A2b) to Q1 (A 1 b) 
of $14 \%$. Bursts were not calculated. The lysogenization of Q1 by A1 $b$ at the same phage: bacteria ratio is $\mathbf{4 5} \%$.

The batch of $A 2 b$ used for the second half of this interaction, when titrated on Q1, had a plaque count of $2 \cdot 6 \times 10^{10} / \mathrm{ml}$. On Q1 (A1b) the plating efficiency was greatly reduced, and the plaques were so shallow and small that an accurate count was impossible; from the results given by titration in decimal dilutions, it was estimated to be about $10^{6} / \mathrm{ml}$. Adsorption at a 1:1 ratio was of the usual order and was followed by only trivial phage multiplication (Fig. 6). Opacity curves (Fig. 7) of cultures of Q1 (A $1 b)$ exposed to A $2 b$ confirmed the poor plating efficiency. At the

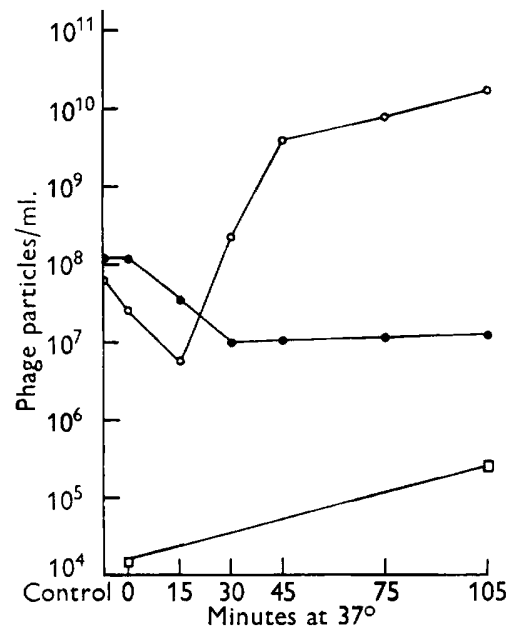

Fig. 6

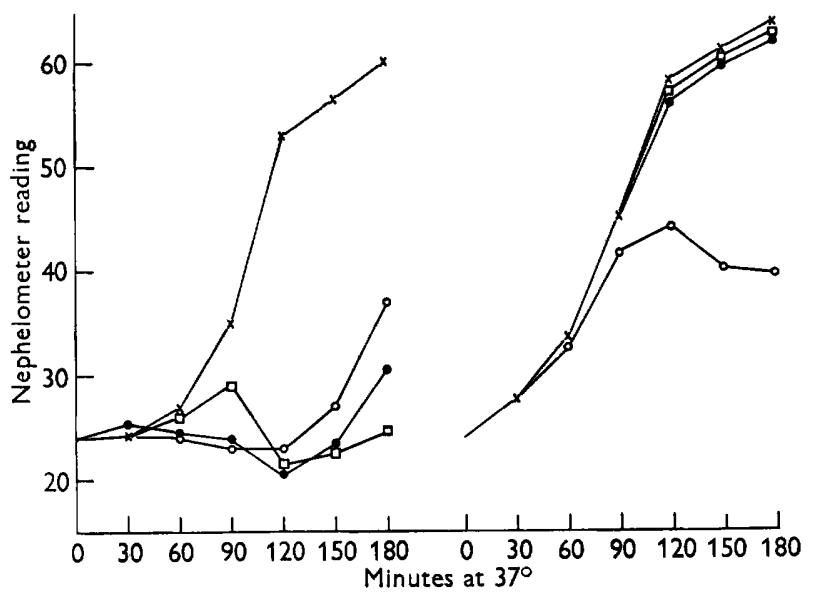

Fig. 7

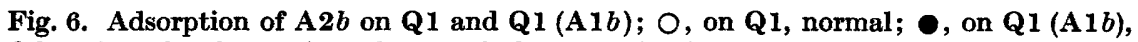
delayed, and only a minor degree of phage production; $\square, A 1 b$ in control culture of Q1 (A1b).

Fig. 7. Opacity curves of $\mathrm{Q} 1$ and $\mathrm{Q} 1(\mathrm{A1} 1 b)$ exposed to A2b. A2b was actively lytic against Q1. Against $Q 1(A 1 b)$ the lytic action was seen only in the 10:1 ratio after 120 min. $x$, control; $0,10: 1$ ratio; $0,1: 1$ ratio; $\square, 0 \cdot 1: 1$ ratio.

lower ratios $(0 \cdot 1: 1$ and $1: 1)$ the curves corresponded closely to the control and showed no clearing. At the 10:1 ratio clearing occurred between 120 and $150 \mathrm{~min}$., indicating that at this phage concentration some vegetative development occurred. Attempts to demonstrate conversion by method 3 were unsuccessful; even at a 10:1 ratio all the colonies recovered were unchanged Q1 (A1 b). Using method 1, 57 colonies of normal appearance were selected and tested. Forty-three were Q1 (A2b), 13 were Q1 (A1b) and one was 'cured'. No attempt was made to estimate the number of bursts, though the opacity curves show that lysis occurred. This is one of the cases in which, despite the negative results given by method 3 , the conversions by method 1 in repeated experiments were unexpectedly high.

To summarize, A1 $b$ evicted and replaced prophage A2b in Q1 (A2b), but only to about 1/3 the extent to which it affected Q1 (A 1 $a$ ). A2 $b$ had a similar but weaker action against $\mathrm{Q} 1(\mathrm{~A} 1 b)$. In both cases 'cures' occurred.

$A 1 \mathrm{~b} \rightleftharpoons A 1 \mathrm{c}$. Neither of these phages had any visible lytic action on the opposite lysogenic organism. The opacity curves showed some retardation in the higher 
phage concentrations, but no clearing. In both cases adsorption was $90 \%$ or over at a $1: 1$ ratio (Figs. 8, 9). Despite the absence of visible lysis, in both cases the rise in the free-phage titre, after a lag period of $45 \mathrm{~min}$. or longer, showed that bursts had occurred, while the increase of free phage from the prophage of the lysogenic organism indicated that there had been some induction. Attempts to demonstrate prophage substitution by method 1 revealed a conversion rate of $1 / 20$ when $Q 1(A 1 c)$

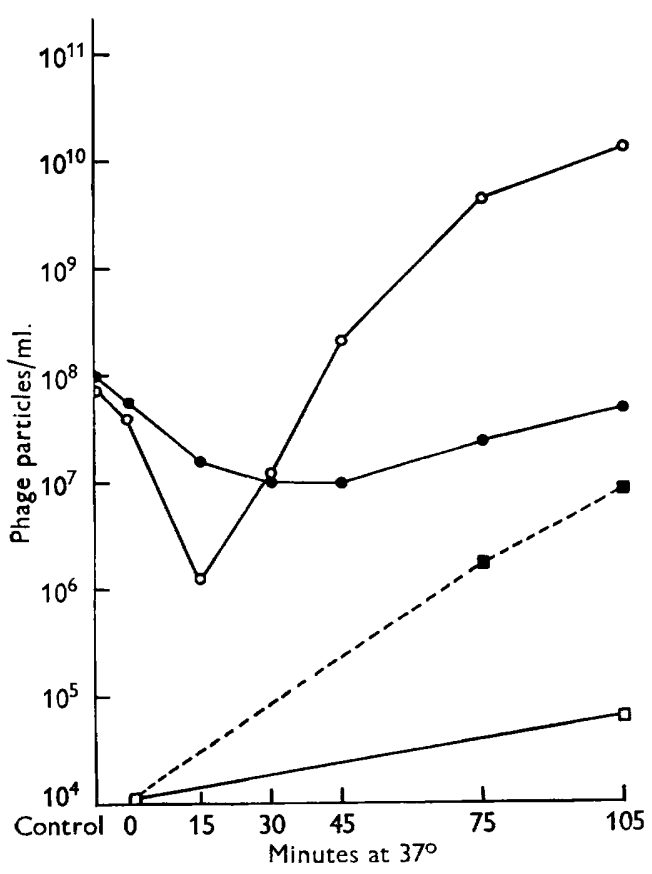

Fig. 8

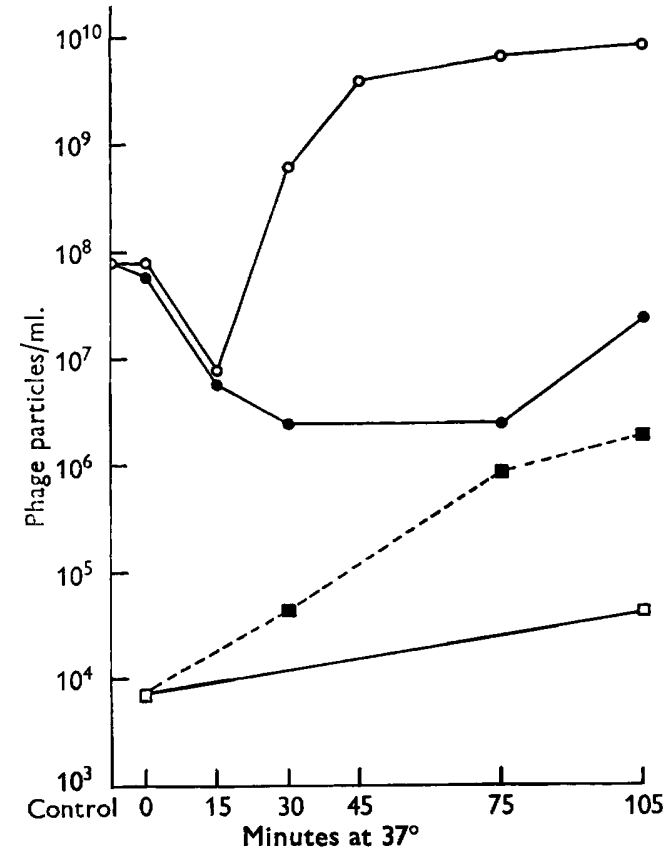

Fig. 9

Fig. 8. Adsorption of A1b on Q1 and Q1 (A1c). Adsorption on Q1 (A1c) was more limited than in the control Q1. Free phage production was registered after 45 min. incubation. There was some induction of $Q 1(A 1 c)$. $O, A 1 b$, adsorbed on $Q 1$; $O$, on Q1 $(A 1 c)$. $\square$, Free phage from induction of $Q 1(A 1 c)$. $\square, A 1 c$ in control culture of Q1 (A1c).

Fig. 9. Adsorption of A1c on $Q 1$ and $Q 1(A 1 b)$. Adsorption on $Q 1$ and subsequent phage production was normal. Adsorption on Q1 (A $1 b$ ) was normal, but phage production was delayed. $\mathrm{Q} 1(\mathbf{A} 1 b)$ was induced to a limited extent. $0, \mathbf{A} 1 c$, Adsorbed on Q1; $\bullet$, A $1 c$ adsorbed on Q1 (A1b); $\mathbf{D}, \mathbf{A} 1 b$ from induction of Q1 (A1b); $\square, A 1 b$ in control culture of Q1 $(\mathbf{A} 1 b)$.

was superinfected with $\mathrm{A} 1 b$, and 16/20 in the reverse reaction, $\mathrm{A} 1 c$ against $\mathrm{Q} 1(\mathrm{~A} 1 b)$. However, using method $3, A 1 c$ at a 10:1 ratio failed to effect prophage substitution in $\mathbf{Q 1}(\mathrm{A} 1 b)$.

\section{Double lysogenization}

$A 2 \mathrm{e} \rightleftharpoons A 1$ a. A2 $e$, which has much the same spectrum of lytic action as $\mathrm{A} 1 b$, gave double lysogenization with Q1 (A $1 a)$. The plating efficiency was not significantly lower on Q1 (A $1 a$ ) than on Q1, the plaque counts of the batch of phage used in these experiments being $4 \cdot 4 \times 10^{9} / \mathrm{ml}$. and $5 \cdot 0 \times 10^{9} / \mathrm{ml}$. respectively. Adsorption 
of A2 $e$ on Q1 (A1a) was as active and rapid as on Q1 (Fig. 10), but in both cases free phage production was delayed. The opacity curves of infected cultures revealed lysis in the lower concentrations, which was more marked in the Q1 cultures than in $\mathbf{Q} 1(\mathrm{~A} 1 a)$, particularly in the $0 \cdot 1: 1$ ratio.

As the Levine technique cannot be used in double lysogenizations, the degree of double lysogenization and lysis resulting from the superinfection of Q1 (A 1 a) with

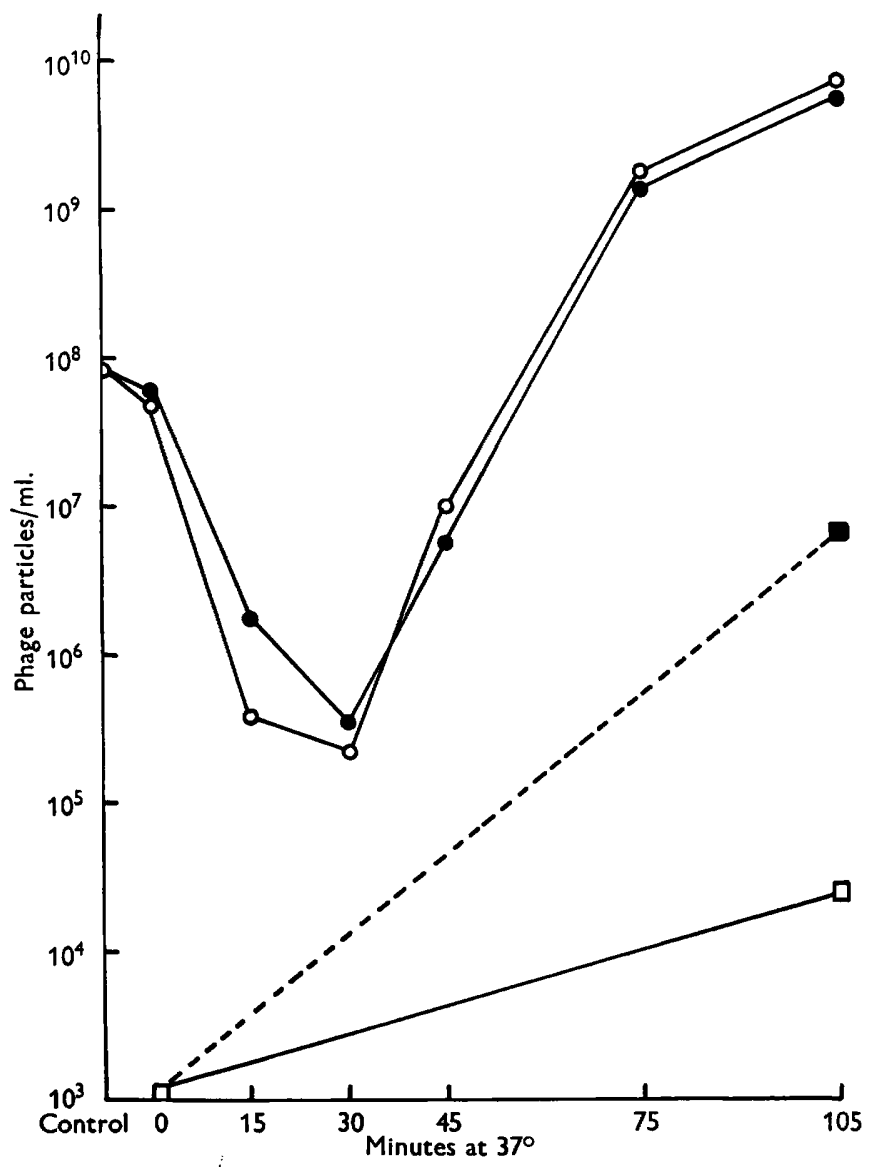

Fig. 10. Adsorption of A2 $e$ on Q1 and Q1 (A1 $a)$. There was no significant difference between the two curves. Adsorption was active, free phage production slightly delayed. There was some induction of Q1 (A1 $a$ ). O, Adsorption of A2e on Q1; , on Q1 (A1 $a$ ); 口, A $1 a$ from induced Q1 (A1 $a$ ). $\square, A 1 a$ in control culture of Q1 (A1a).

A2 $e$ was determined by method 3. The results are recorded in Table 4, where they are compared with the corresponding figures given by Q1 infected with the same batch of A 2e. In the lower ratios, there was no significant difference. In the 10:1 ratio there were fewer bursts, and more lysogenizations on Q1 than there were bursts and double lysogenizations on Q1 (A 1a). Boyd \& Bidwell (1961) showed that A $2 e$ produces a lower percentage of lysogenizations in Q1 than does A1 $b$. Taking this into consideration, $A I b$ and $A 2 e$ are alike in the way they affect 
Q1 (A $1 a$ ), except that A $1 b$ gives prophage substitution and A $2 e$ double lysogenization. Prophage Al $a$ confers on its host bacterium little if any immunity against either of these superinfecting phages. In the reverse reaction, although there was no visible lytic action, A1 $a$ was well adsorbed to Q1 (A 2e), about $95 \%$ being removed in $30 \mathrm{~min}$., from a mixture having a $1: 1$ ratio. There was no evidence

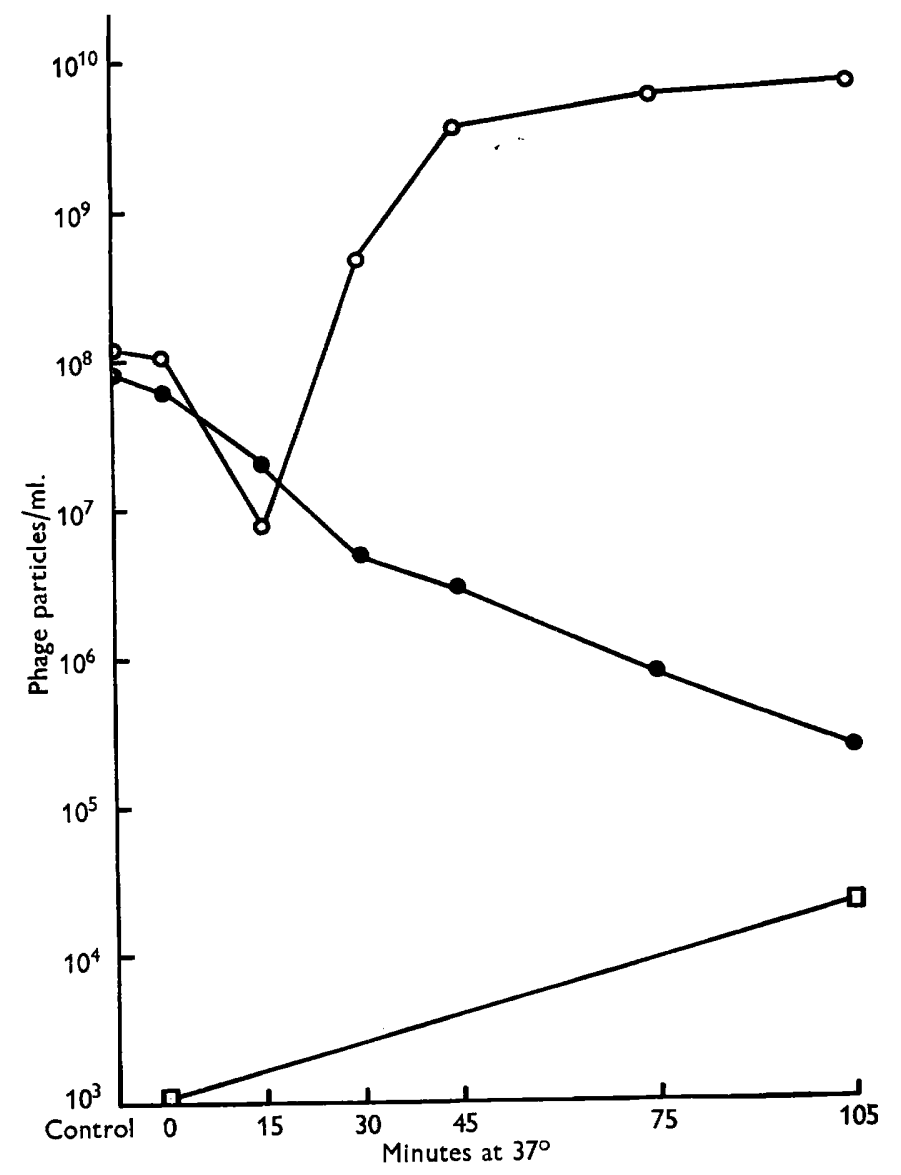

Fig. 11. Adsorption of A1 $a$ on Q1 and Q1 (A2e). Adsorption was delayed. There was no phage production. $\mathrm{O}$, On $\mathrm{Q} 1$;, , on $\mathrm{Q} 1(\mathrm{A2} e)$; $\square, \mathrm{A2} e$ in a culture of $\mathrm{Q} 1$ (A2e).

Table 4. Comparison of $Q 1$ infected and $Q 1$ ( $A 1$ a) superinfected with $A 2 \mathrm{e}$

As in Table 3, except that the technique of method 3 was followed. In the 0.1:1 ratio of superinfection no conversions of Q1(A1 $a$ ) were found in 128 colonies examined. A2e was the only phage present in the bursts.

Phage : bacteria ratio

Phage A2 $e$ infecting or superinfecting

Lysogenics

Bursts

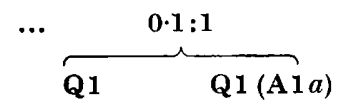

$\mathrm{Q1}(\mathrm{A1} a) \overparen{\mathrm{Q1}}$

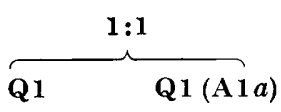

Q1 (A $1 a)$

11.3

30

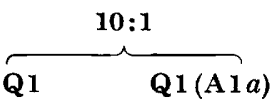

57-7 4.4
36

8 
of vegetative development in the later stages of this experiment (Fig. 11). Using method 1, 40 colonies were examined; only 3 showed double lysogenization. This reaction, though present, was therefore weak. In this and other similar experiments with these phages no cures were found.

$A 1 \mathrm{~b} \rightleftharpoons A 2 \mathrm{c}$. A1 $b$ and A2 $c$ each had a weak lytic action on the opposite lysogenic organism. The plating efficiency of A $1 b$ on Q1 (A 2c) was considerably less than on $Q 1$. Thus a batch having a plaque count of $1.6 \times 10^{10} / \mathrm{ml}$. on $\mathrm{Q} 1$ gave a

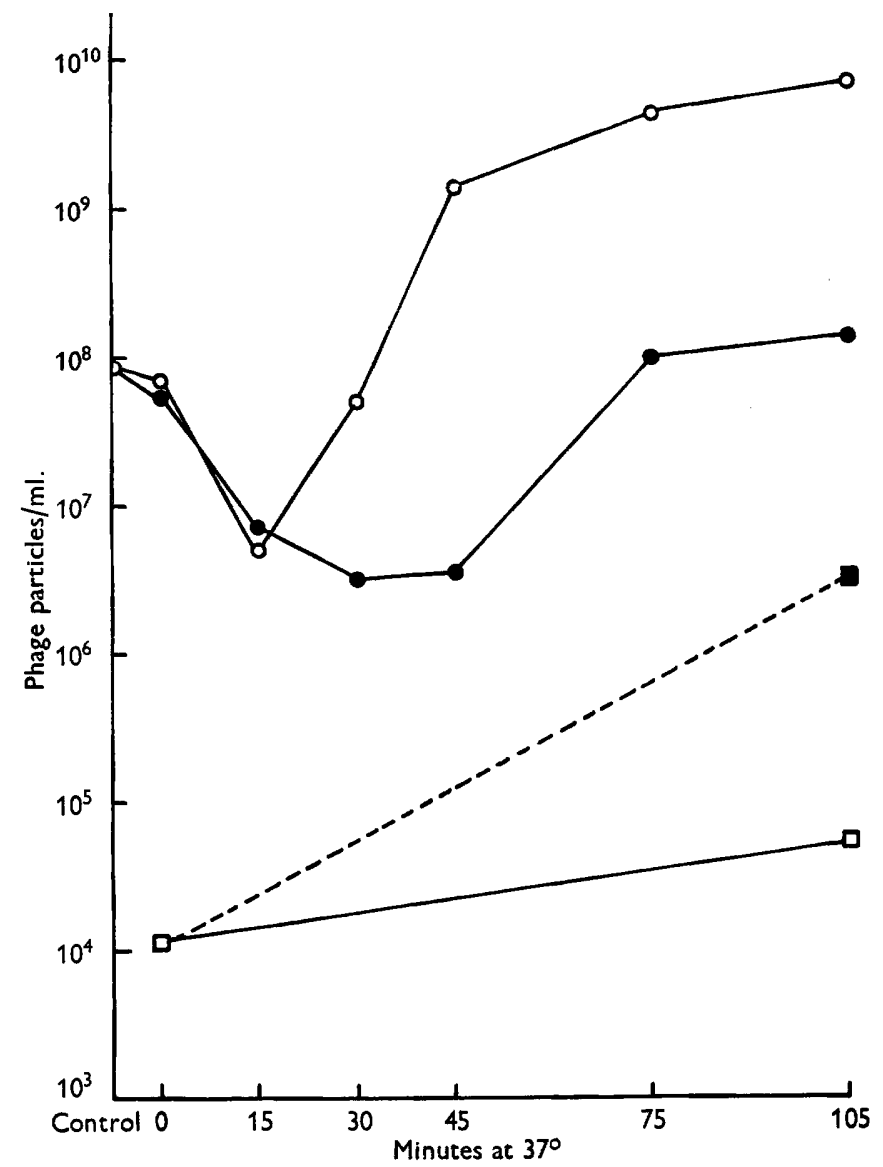

Fig. 12. Adsorption of $A 1 b$ on $Q 1$ and $Q 1$ (A2c). Adsorption on $Q 1$ (A2c) was as active as on Q1, but phage production was delayed for $45 \mathrm{~min}$. Q1 (A2c) showed some induction. $O$, Adsorption of $\mathrm{A} 1 b$ on $\mathrm{Q} 1$; $\boldsymbol{O}$, adsorption of $\mathrm{A} 1 b$ on Q1 (A2c); $\mathbf{A}, \mathbf{A 2 c}$ from induction of Q1 (A2c); $\square, A 2 c$ in control culture of Q1 (A2c).

count of $7.7 \times 10^{7} / \mathrm{ml}$. on $\mathrm{Q} 1$ (A 2c). The plaques on Q1 (A 2c) were shallow, and varied considerably in size, some being so small that they could only be seen by transmitted light, using a plate microscope. Adsorption was similar to that on Q1, but phage production did not occur for $45 \mathrm{~min}$. There was some induction of Q1 (A 2c) (Fig. 12). Opacity curves of Q1 (A2c) superinfected with A1b showed some retardation of growth but no clearing in the 10:1 and 1:1 ratios; in the $1: 10$ 
ratio slight clearing occurred after $150 \mathrm{~min}$. incubation. In a superinfection experiment, carried out by method 3 at a $1: 1$ ratio, $2.5 \%$ of the Q1 (A 2c) bacteria were doubly lysogenized, i.e. converted to Q1 (A1b, A2c). Bursts were not estimated and no cures were found in $\mathbf{1 3 0}$ colonies which were examined. In view of the poor plating efficiency the relatively high conversion rate is notable. Method 3 gave better results than method 1 (compare Q1 (A1b) superinfected by A2b).

The plating efficiency of A2c on Q1 (A1 b) was considerably higher than in the reverse reaction. The plaques were nevertheless much smaller (half size or less) and shallower than corresponding plaques on $\mathrm{Q} 1$. The respective plaque counts of the batch tested were $1.44 \times 10^{10} / \mathrm{ml}$. on $\mathrm{Q} 1$, and $1 \cdot 14 \times 10^{9} / \mathrm{ml}$. on $\mathrm{Q} 1(\mathrm{Al} b)$. Adsorption was good, being little different from adsorption on Q1, but, as would

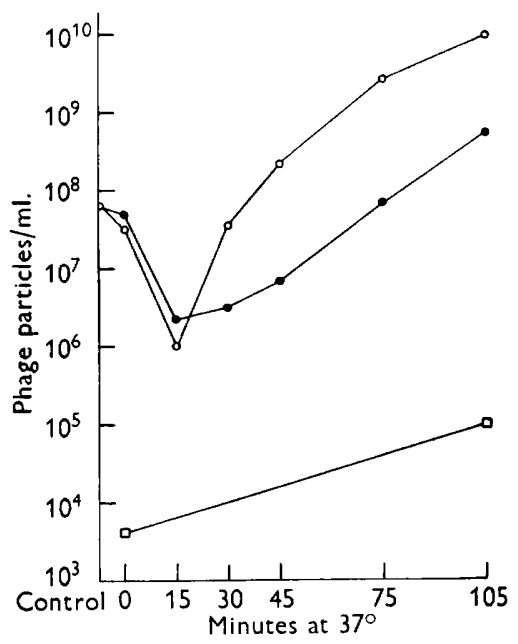

Fig. 13

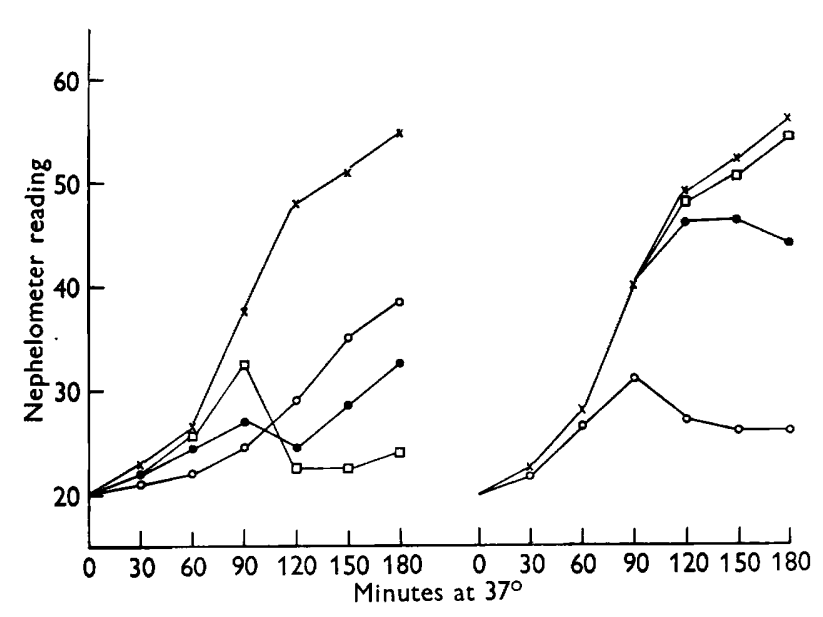

Fig. 14

Fig. 13. Adsorption of A2c to Q1 and Q1 (A1b). Adsorption on Q1 (A1b) was similar to the control, but free phage production was delayed. $O$, Adsorption of A2c on Q1; $\odot$, adsorption of $A 2 c$ on $Q 1(A 1 b) ; \square, A 1 b$ in control culture of $Q 1(A 1 b)$. There was no significant induction of $\mathrm{A} 1 b$.

Fig. 14. Opacity curves of cultures of $Q 1$ and $Q 1(A 1 b)$ exposed to A2c. Against Q1 $(A 1 b), A 2 c$ had no action at $0.1: 1$ ratio, showed delayed clearing at $1: 1$, and at 10:1 gave clearing similar to $0 \cdot 1: 1$ in the control against $Q 1$. $\times$, Control; $O, 10: 1$ ratio; , $1: 1$ ratio; $\square, 0 \cdot 1: 1$ ratio.

be expected, subsequent phage production was less active (Fig. 13). The opacity curves (Fig. 14) showed clearing in the 10:1 and 1:1 ratios. Using method 3 at a $1: 1$ ratio, no prophage change was detected: at $10: 1,13.6 \%$ of the bacteria were doubly lysogenized. No cures were found.

$A 1 \mathrm{~b} \rightleftharpoons A 2 \mathrm{e}$. The two phages $\mathrm{A} 1 b$ and $\mathrm{A} 2 e$, both active against several other lysogenic strains, were cross-immune in terms of detectable lytic action, but when examined by method 1 , each phage was found to produce double lysogenization in the opposite lysogenic strain, though A2e as a superinfecting phage was more 
active than $A 1 b$. In a mixed culture of the doubly lysogenic strains and $Q 1$, the predominant free phage, irrespective of the origin of the strain (whether $A 1 b$ on Q1 (A $2 e$ ) or vice versa) was Alb. In early experiments the presence of A2e was missed because the propagating culture was not incubated for a sufficiently long time.

\section{Interaction of phages of different antigenic structure}

Ten of the twelve phages under consideration - those of the A1 and A2 serieshave a similar antigenic structure, while A3 and A4 have distinctive antigens, and also differ from the $\mathbf{A} 1$ and $\mathbf{A} 2$ phages in that they markedly reduce the ca pacity of the bacteria they lysogenize to adsorb homologous and heterologous phages

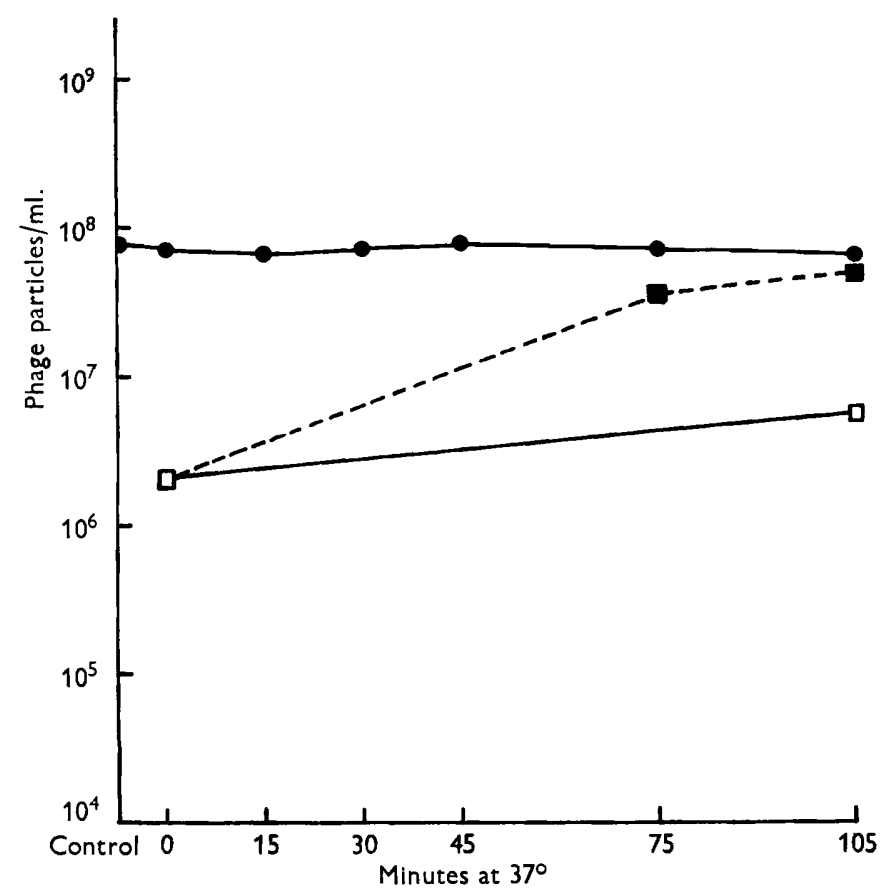

Fig. 15. Adsorption of A3 on Q1 (A1b) and of A1b on Q1 (A3). A3 was freely adsorbed on Q1 (A1b) without any production of free phage. Adsorption of A1b on Q1 (A3) was too trivial to be detectable by this technique, yet after an interval of $45 \mathrm{~min}$. free phage was produced, showing that adsorption and lysis had occurred., A3 on Q1 (A1b); O, Alb on Q1 (A3).

(Boyd, 1954). This was less obvious in the case of Q1 (A3) which despite its poor adsorptive properties showed, when superinfected with the other phages, a good spectrum of lysis with a more limited degree of prophage change. In Fig. 15 the adsorption of A1 $b$ on Q1 (A3) is shown in comparison with the reciprocal reaction. The latter followed the normal pattern, while in the former there was no detectable reduction of free phage particles, and the only evidence of adsorption was their increase after an interval of $45 \mathrm{~min}$., presumably the outcome of productive development. Other members of the group reacted in the same way, as can be seen in respect of $\mathrm{A2} b, \mathrm{A2} d$ and A2 $e$ in Fig. 16. The visible lysis produced by 
method 1 was well marked, and in comparison with this, prophage change was weak. Table 5 gives the percentages of bacteria showing prophage change when superinfected in a 10:1 ratio, using method 3.

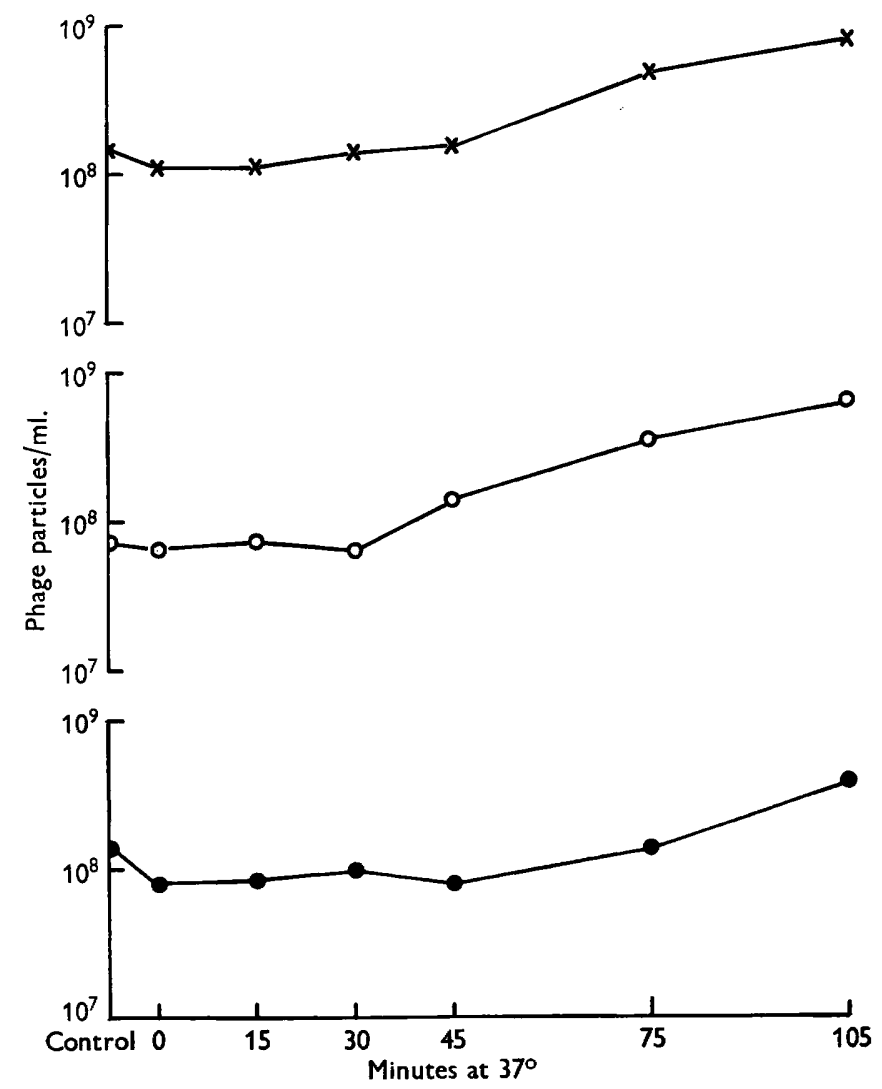

Fig. 16. Adsorption of A2b, A2d, and A2e on Q1 (A3). All three were feebly adsorbed, but gave delayed liberation of free phage. $\times, \mathbf{A 2} b ; 0, \mathbf{A 2 d} ; 0, \mathbf{A 2} e$.

Table 5. Prophage change in superinfected Q1 (A3)

The technique of method 3 was followed.

Superinfection of $Q 1$ (A3) in a 10:1 ratio by phages

Percentage of

Q1 (A3) showing

prophage change

$\begin{array}{ccccccccccc}\text { A1 } a & \text { A1 } b & \text { A1 } c & \text { A1 } d & \text { A2a } & \text { A2b } & \text { A2f } & \text { A4 } & \text { A2c } & \text { A2d } & \text { A2e } \\ 0 & 3.6 & 0 & 2 & 2.4 & 0.7 & 0 & 0 & 3.4 & 1.25 & 6.6\end{array}$

The characters present in A3 are more strongly marked in A4. Selecting as an example its reciprocal action with A1b, A4 was freely adsorbed to Q1 (A $1 b$ ) whereas the adsorption of A1b to Q1 (A4) was trivial (Fig. 17), and was followed by only a feeble rise in titre which is in general agreement with the weak lytic 
action shown in Fig. 1. We have failed in repeated tests to provoke prophage substitution in Q1 (A4) by superinfection with A1b. Conversely A4, though producing no visible lysis in Q1 (A1 $b$ ), was capable of evicting and replacing its prophage.

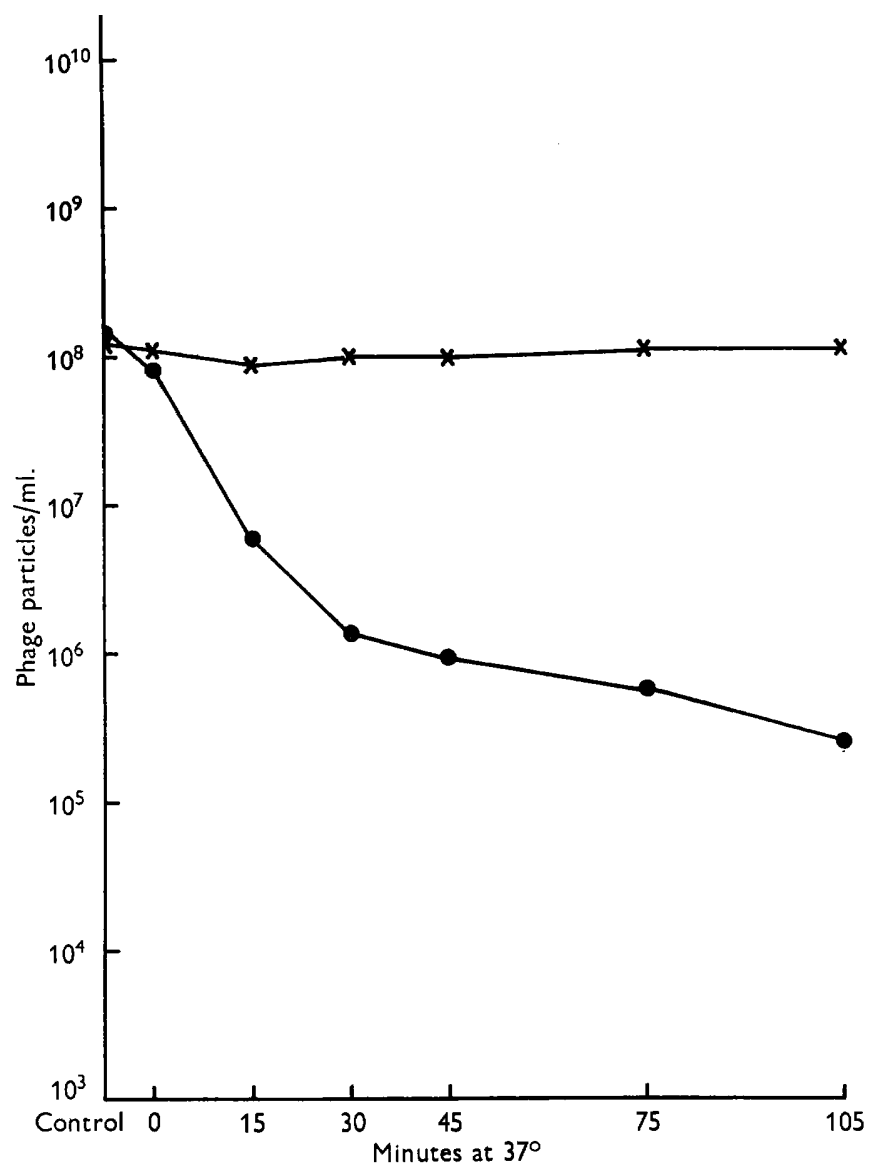

Fig. 17. Adsorption of A1b on Q1 (A4) and A4 on Q1 (A1b). A4 was freely adsorbed on $\mathrm{Q} 1$ (A1b), without production of free phage. A1 $b$ was weakly adsorbed on $\mathbf{Q} 1$ (A4), with slight production of free phage. $\times, A 1 b$ on $Q 1$ (A4);,$A 4$ on $Q 1$ (A1b).

Superinfections of Q1 (A4) with A1 b, A2c, and A2d were the only instances encountered in this series in which lysis occurred unaccompanied by detectable prophage change. It was deemed of interest to determine whether the superinfecting phage was multiplying in Q1 (A4) or was inducing prophage A4, and burst experiments were carried out with A1 $b, \mathrm{A2} c, \mathrm{A2} d$ and also A2 $e$ (Table 6). Apparently these superinfecting phages may either multiply in Q1 (A4) without inducing the prophage, or conversely may induce the prophage without themselves multiplying. In a few instances both phages have been found in the same tube, but as some of the tubes must have contained more than one bacterium, it cannot be concluded that in such cases both phages multiplied together in one host organism. The plaque 
characters of A4 are distinctive, and consequently the identification of the two types presented no difficulty. In only one of the bursts was there evidence of hybridization.

The only instance of prophage substitution in Q1 (A4) was in superinfection with A 2 $b$, where two colonies of Q1 (A 2b) were found in sixty examined.

The action of A2 $e$ on Q1 (A4) was of especial interest. A2 $e$ had a spectrum of lytic activity closely resembling that of $\mathrm{A} 1 b$. It was aggressive in producing lysis and lysogenesis, and in its prophage phase afforded a good degree of protection against lysis by the other members of the series, but less against prophage change, which in the appropriate grouping occurred either as substitution or as double lysogenization. However, A2 $e$ differed from A $1 b$, and indeed from all the other phages of the group, in that it had a well-marked lytic action on Q1 (A 4), mainly due to induction (Table 6 and Fig. 18), and also produced a considerable degree of double lysogenization. This was not associated with better adsorption (Fig. 18, compare with Fig. 17).

Table 6. Superinfection of $Q 1(A 4)$ : analysis of bursts

Superinfection

A1b on Q1 (A4)

\section{Phage:} bacteria
ratio

$1: 1$

$1: 1$

$4: 1$

A2c on Q1 (A4)

A2d on Q1 (A4)

A2e on Q1 (A4)
Estimated bacteria per tube

1 bacterium in 4 tubes

1 bacterium in 1 tube
Nos. of plaques in bursts

\begin{tabular}{|c|c|c|}
\hline$A 1 b$ & A4 & \\
\hline 78 & - & \\
\hline 120 & - & \\
\hline & 244 & \\
\hline 120 & - & \\
\hline 4 & 176 & \\
\hline 100 & - & \\
\hline 134 & - & \\
\hline 240 & - & \\
\hline 12 & 188 & \\
\hline 58 & 50 & \\
\hline 160 & - & \\
\hline- & 168 & \\
\hline- & 64 & \\
\hline$\overline{4}$ & 164 & \\
\hline - & 62 & \\
\hline 2 & 84 & \\
\hline- & 56 & \\
\hline $\mathbf{A} 2 c$ & A4 & \\
\hline 976 & - & \\
\hline 204 & - & \\
\hline 284 & - & \\
\hline $\begin{array}{c}\text { A2d } \\
90\end{array}$ & A4 & \\
\hline 4 & 44 & \\
\hline A2e & A4 & ? hybrids \\
\hline 一 & $\begin{array}{l}48 \\
40\end{array}$ & \\
\hline 4 & $\begin{array}{r}40 \\
122\end{array}$ & \\
\hline 8 & 654 & \\
\hline 108 & 12 & 38 \\
\hline
\end{tabular}




\section{Cures}

In carrying out prophage substitution experiments by method 1 , we isolated non-lysogenic, i.e. 'cured', bacteria on a number of occasions, particularly from Q1 $(A 1 b)$. The reactions in which these occurred are not fully recorded in this paper, as the phenomenon has not been investigated with sufficient thoroughness. On only one occasion (Q1 (A4) superinfected by A 2e) did we encounter cure in association with double lysogenization.

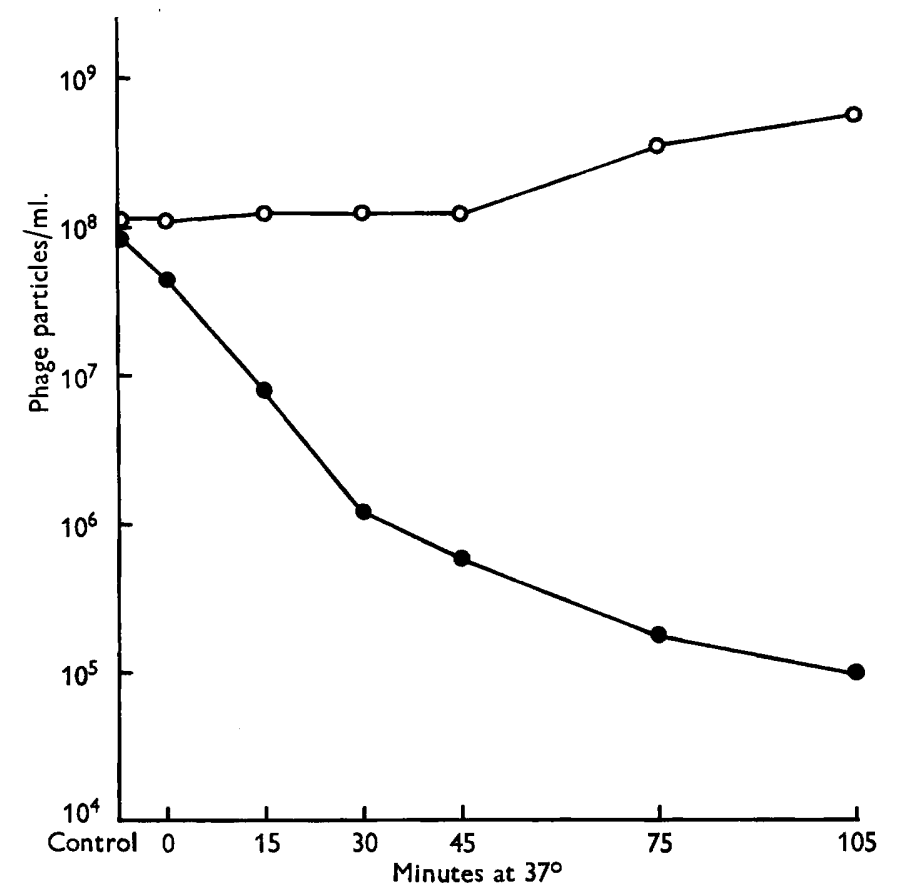

Fig. 18. Adsorption of A2 $e$ on Q1 (A4). The curve gave certain evidence neither of adsorption nor of multiplication of A2e. There was, however, some induction of Q1 (A4). $\checkmark$, A2e on Q1 (A4); $\square$, free phage A4 from induction of Q1 (A4); $\square, A 4$ in control culture of $\mathbf{A} 4$.

\section{DISCUSSION}

The problems of prophage substitution and double lysogenization were first studied by Bertani (1953, 1954) who worked with strains of phage isolated from Escherichia coli $\mathrm{Li}$. In all but his first experiments the 'wild' form of one of these strains, P2, was used together with mutants obtained from it by irradiation*: the mutants could be distinguished from the wild form by their plaque characters. A strain of Shigella dysenteriae (Sh) served as indicator. When Sh (P2) was superinfected with one of the mutants, all the cells survived superinfection, though after a few generations a percentage of superinfected cells 'burst' liberating phages of both types. In certain cases both prophage substitution and double lysogenization were found, while, after several hours incubation, cells were isolated in which

* We have recently been informed by Dr Bertini that in his earlier work the mutants he used occurred spontaneously and not as the result of irradiation. 
segregation had not occurred, and daughter cells containing one or other of the prophages continued to be produced. A few recombinants were detected. Following up this work, Bertani \& Six (1958), using these same phages and another, P2 Hy dis (Cohen, 1959), together with a large number of artificially produced mutant strains of Escherichia coli $\mathbf{C}, E$. coli $\mathbf{K} 12$, and Shigella dysenteriae, found that there were several chromosomal sites to which these phages could become attached, one the preferred location, the others second-choice locations. Six $(1960,1961)$ extended these observations, and found that genetic incorporation occurred most readily in the preferred location, either by prophage addition or substitution, and that the frequency of these changes increased linearly with multiplicity. It is to be noted that these experiments were carried out with mutants of one parent phage; all were therefore very closely related.

Groman (1955) and Groman \& Eaton (1955) experimented with phages recovered from Klebsiella diphtheriae. Their object was to demonstrate the relationship of toxigenicity to lysogenization with one of these phages, but in doing so they produced evidence of prophage substitution, of double lysogenization, of recombination and of 'cure'. Their findings differ from those of Bertani and Six in that all the phages used were 'wild'. Gorrill (1957) reported prophage substitution and double lysogenization with staphylococcal phages. Zinder (1958) studied lysogenization and superinfection immunity in Salmonella but did not specifically investigate prophage substitution and double lysogenization. He found that when a prophage did not confer immunity to superinfection with a related phage, the same general effects occurred as those following infection of a non-lysogenic strain. He suggested that the first step is comparable to U.V. induction.

The observations recorded in this paper, on which a brief preliminary note was published (Boyd, 1956), differ from those of other workers in that they concern a relatively large group of closely related phages, all of which, apart from adaptation to a common host bacterium, are in the 'wild' state (Boyd \& Bidwell, 1957). To what extent they are descended from a common source can only be a matter for speculation. They were found in lysogenic strains of Salmonella typhimurium isolated in different laboratories throughout the British Isles from faeces, contaminated food, etc. A few came from overseas, and there is little doubt that these and other related phages have a world-wide distribution. The prophage change which has been observed in laboratory experiments may well occur in natural surroundings if lysogenic bacteria carrying different types of phage are brought into close proximity, and in this way new types may arise from the emergence of recombinants.

Although the end-result of superinfection depends on the properties of both superinfecting phage and lysogenic bacterium, a better idea of the part played by each of the participants in the reaction is to be obtained by considering them separately. 


\section{The varying properties of the superinfecting phage}

Aggressive and non-aggressive phages. There was considerable difference in the aggressiveness of the different phages (Fig. 1, Table 1). Some (A1b, A1c, A2e), appeared to dominate many of the lysogenic strains, and brought about well-marked lysis and prophage change; others (A $1 a, \mathrm{~A} 2 f, \mathrm{~A} 3$ ) were, in terms of lysis, apparently inactive, and produced only feeble prophage change; the remaining six occupied intermediate positions. This pattern of action was not consistent, however, and sometimes the so-called weak phage gave a strong reaction where a strong phage reacted weakly. Thus A2b and A2d produced well-marked lysis of Q1 (A2c), against which the aggressive $A 1 c$ and A $2 e$ were inactive, whilst $A 1 b$ gave only faint lysis. A2b lysed Q1 (A1c), A $1 b$ did not. A2c lysed Q1 (A1b), A1c did not. Other minor examples can be seen in Fig. 1.

Relationship of lysogenizing and lytic action. In the early stages of this work it was assumed that prophage change would be found in lysogenic bacteria superinfected with heterologous phage only where visible evidence of coincident vegetative development was demonstrated by the formation of plaques. Doubt was cast on this assumption by the discovery that in some cases (e.g. Q1 (A 1 b) superinfected with A 2c) the degree of prophage change bore no relation to the plaquing efficiency of the particular phage on the lysogenic organism. In certain pairs (e.g. A $1 a$ and A $1 b$ ) in which the cross-immunity test, based on plaquing, gave a one-way reaction, it could be shown that the prophage change was two-way, although much less active in the lysogenic strain which appeared resistant to lysis by the opposite phage. Observations were then extended to pairs which, in terms of plaquing, seemed to show complete cross-immunity. It was found that a limited degree of prophage substitution or double lysogenization sometimes occurred. If a sufficiently exhaustive examination were made, some prophage change could probably be detected in many of the interactions now recorded as negative in Fig. 1. Nor can the absence of visible plaque formation always be taken as proof that there has been no vegetative development either of the superinfecting phage or of induced prophage. Trivial degrees of prophage change can be detected by method 1, but we have no corresponding simple technique to detect minor degrees of vegetative development, though the late upward trend of the phage titre in some adsorption tests (Figs. 8, 9) indicated that lysis had occurred.

Superinfecting phages which gave well-marked lysis were usually active in producing prophage change while non-aggressive ones were not. Thus the aggressive phages A1 $b, \mathbf{A} 1 c, \mathbf{A} 2 e$, and to a lesser extent A2b and A2d, which had a wide range of lytic activity, effected a considerable degree of prophage change. To this generalization there were exceptions, e.g., in superinfection of Q1 (A1c) with A2b, there was a moderate but constant degree of lysis together with an almost complete absence of prophage change. Conversely, the non-aggressive phages Al $a$, A $2 f$ and A3 which with one minor exception showed no lytic activity, effected very little prophage change. 


\section{The significance of the antigenic structure of superinfecting phage}

The antigenic structure of the phage had no obvious bearing on its superinfecting capacity. A3 was found to have a spectrum of action very similar to that of A1 $a$ and A $2 f$, while A4 had weak activity not unlike A1d and A2a.

\section{Evidence of different sites of attachment to the chromosome}

The most striking feature of these findings was the sharply defined pattern of prophage substitution and double lysogenization which split the series into two groups (Fig. 1). The larger, group 1, comprised A1 $a, \mathbf{A 1} b, \mathbf{A l} c, \mathbf{A 1} d, \mathbf{A 2} a, \mathbf{A 2} b$, A2 $f$, A3 and A4. The smaller, group 2, comprised A2c, A2d, and A2e. The type of prophage exchange between any two members of group 1 or any two members of group 2, with exceptions which do not affect the significance of the observation, was prophage substitution. When lysogenic strains of group 1 were superinfected with phage of group 2, or vice versa, double lysogenization resulted. This amply confirmed observation suggests that there are two sites on the bacterial chromosome to which the infective element of these phages can become attached as prophage, and that each group is restricted to one of these sites. Thus superinfecting phages of either group were able to establish themselves in heterologous lysogenic strains of the same group only by dislodging the resident prophage. On the other hand, superinfecting phages of group 1 , by attaching themselves to the vacant site in the lysogenic bacteria of group 2, produced double lysogenization, while group 2 phages reacted similarly with group 1 bacteria. There was one apparent deviation from this rule which requires some explanation. As superinfecting phage, A2 $f$ behaved as a member of group 1 : as prophage in Q1 (A2 $f$ ) it was evicted and replaced by superinfecting phages of both groups except on one occasion, when superinfection with A2d gave double lysogenization. However, the double lysogenizations which superinfections with A2 $f$ produced in members of group 2 showed clearly that A $2 f$ must occupy a different site from the group 2 prophages. A possible explanation of the anomalous findings is that, while A2 $f$ as an invading phage could establish itself on the vacant site, as resident prophage its attachment was insecure, so that it was displaced by the invading phages as they became attached to the alternative site. Another possibility is that prophage A2f occupied a slightly different site from the other group 1 prophages, in closer proximity to the group 2 site, and because of this was more liable to be dislodged.

The varying properties of the lysogenic bacterium (bacterium-prophage complex)

Variations in the degree of immunity. In general terms, the aggressive phages (A $1 b, \mathbf{A} 1 c, \mathbf{A} 2 e$ ), when present in the host bacterium as prophage, offered a considerable degree of resistance to most superinfecting phages, and to that extent conferred immunity to both lysis and lysogenesis (see Fig. 1, Table 1). On the other hand, the non-aggressive phages (A $1 a, \mathbf{A} 2 f$ and A3) had little or no protective action other than against one another, while strains $\mathbf{A} 1 d, \mathbf{A} 2 a, \mathbf{A} 2 b$ of group 1 were intermediate. A4 is in a category by itself, and will be discussed separately. The two remaining intermediate strains, A2 $c$ and A2d, which belong to group 2, produced, as prophages, a wider range of immunity than did the corresponding strains in group 1 . 
The results given by superinfection with $\mathrm{A} 1 b$ of all the lysogenic strains (Fig. 1, Table 2) provide a detailed example of the varying degrees of immunity to one particular phage which the different prophages can confer. Resistance to prophage change when exposed to a 1:1 ratio varied from $100 \%$ in Q1 (A1c), Q1 (A4), Q1 (A2d) and Q1 (A2e) to nil in Q1 (A1 $a)$, which exhibited no resistance of any kind and gave 'multiplicity infection' results corresponding closely to those given by Q1 (Table 3). This strain was equally susceptible to superinfection with A2e (Table 4).

The most striking feature overall was the irregularity of the reactions. Each lysogenic strain had its own idiosyncrasies.

\section{Variations in the type of resistance offered by different lysogenic bacteria}

Usually, but not invariably, resistance to lysis and resistance to prophage change were roughly parallel. However, resistance to lysis was sometimes greater than resistance to prophage change, when both were considerably reduced (Fig. 1, Table 1). In this respect the reactions of $Q 1(A 1 b)$ and $Q 1(A 1 c)$ are of interest. Both were strongly resistant to lysis though each showed two minor breakdowns. But whereas Q1 (A 1c) resisted prophage change completely in 7 and almost completely in a further 3 of the 11 superinfections, Q1 $(\mathrm{A} 1 b)$ was relatively susceptible, and its prophage was evicted and replaced even by the non-aggressive phages $\mathbf{A} 1 a, \mathbf{A} 2 f$, and A3. The vulnerability of Q1 (A 1 b) to these three phages is the more remarkable when it is recalled that they were cross-resistant to each other. It is of interest to recall that Q1 (A1b) and Q1 (A 1 c) differ in their response to u.v. irradiation (Boyd \& Bidwell, 1959). The reaction of Q1 (A1 $b$ ) when superinfected with A $1 a$ is a good example of immunity to lysis but not to prophage change (Table 1, Fig. 4, and text). A $2 e$, the third of the aggressive phages, behaved in much the same way as A1b, giving a good degree of protection against lysis but less against prophage change.

In contrast, resistance to prophage change can exceed resistance to lysis. This is seen in the reactions of Q1 (A3) and Q1 (A4), which, it will be noted, were completely cross-resistant to superinfection with their reciprocal phages. Apart from immunity to the non-aggressive phages A2 $a$ and A2 $f$, Q1 (A3) showed well-marked lysis with only a limited degree of prophage change when superinfected with the remaining 8 phages (Fig. 1, Table 5). In Q1 (A4) resistance to both lysis and prophage change was greater than in Q1 (A3) and in 3 instances lysis occurred without any accompanying prophage change. Q1 (A3) and Q1 (A4) were alike in having very limited powers of adsorption (Figs. 15, 16, 17, 18), presumably because the prophage interferes in some way with the genetic mechanism which controls the bacterial surface. As a sequel to poor adsorption, the multiplicity effect (Boyd, 1951) must be partly nullified, with a consequent bias towards vegetative development. It is unlikely, however, that this plays a significant part in producing the unusual results. It does not account for the marked difference between the reactions of Q1 (A3) and those of Q1 (A4) in both of which adsorption was equally restricted, nor for the reactions of Q1 (A4) when superinfected with A2e. A2e was no better adsorbed by Q1 (A4) than the other phages of the series, yet it produced wellmarked lysis and a good degree of double lysogenization. This suggests the existence of a barrier or repressor, distinct from defective adsorption, which A2 $e$, but not the others, was able largely to evade. The presence of such a repressor, more active 
in $\mathrm{Q} 1$ (A4) than in Q1 (A3) and directed more against prophage change than against vegetative development, is a plausible if hypothetical explanation of the phenomenon.

Q1 (A 1c) was completely resistant to A2c. This, apart from the reactions of Q1 (A 2f), which have already been discussed, was the only instance of resistance to prophage change where double lysogenization would be expected.

In tests using method 1, lysogenic bacteria were exposed to high concentrations of superinfecting phage, and consequently each bacterium was invaded by several, perhaps many phage particles. (This statement does not apply to Q1 (A3) and Q1 (A4) which have restricted powers of adsorption.) Despite this heavy infection, many of the bacteria did not react, and sometimes only a small percentage underwent either lysis or prophage change. What is the cause of this breakdown of immunity in occasional members of a population which is otherwise resistanta non-resistant bacterium, a more than ordinarily aggressive phage particle, or the cumulative effect of multiple infection? The available evidence suggests that it is the last of these possibilities. On several occasions we have found prophage change when using method 1 where repeated tests by method 3 , in which many bacteria were examined, gave negative results. The significant difference between the methods is that in the former there is a heavy multiplicity of infection while in the latter superinfected bacteria adsorb a relatively small number of particles. The property which confers resistance is probably limited, and can be neutralized by weight of numbers. In these experiments the distribution of particles per bacterium varied around a mean, and the most likely explanation of the occasional positive reactions is that the bacteria involved were so heavily infected that the resistance was saturated and one or more particles left to develop unimpeded. This theory leaves unexplained the few cases in which method 3 gave more favourable results than would be expected from the results given by method 1, as in superinfection of Q1 (A 2c) by A $1 b$. This unexpected finding may be related to lysis from without but requires further investigation.

\section{The mechanism of immunity}

The main interest of these observations lies in the information they provide concerning the mechanism of the immunity of lysogenic bacteria to homologous or heterologous phages. There is no convincing evidence that immunity is related to defective adsorption. The non-aggressive phages of group 1, which have minimal lysogenizing and lytic action, were as freely adsorbed as the aggressive phages which produced marked lysis and prophage substitution. Conversely, adsorption to Q1 (A3) and Q1 (A4) was equally restricted whether the superinfecting phage was active or not. Nor can any major role be attributed to interference resulting from the presence of the prophage on the bacterial chromosome in such a position that it prevented the invading heterologous phage from gaining access to this particular and essential location. It is true that, while there were numerous negative findings in the blocks where prophage substitution would be expected to occur, there were very few in the series showing double lysogenization (Fig. 1). This might indicate that there was less opposition to double lysogenization than to prophage substitution-i.e. that it was easier for a superinfecting phage to occupy a vacant chromosomal site than to evict and replace a resident prophage. However, 
the low incidence of lysis and prophage change in most cases where double lysogenization occurred, particularly in the superinfection of group 2 lysogenic bacteria with group 1 phages, showed clearly that the mere existence of a vacant site did not leave the door open for the invader, and that other and more potent forces were concerned in producing immunity.

Jacob \& Campbell (1959), studying phenomena encountered in the zygotic induction of Escherichia coli $\mathrm{K} 12(\lambda)$ by certain mutants of $\lambda$, suggested that a certain region, $\mathrm{C} 1$, of the chromosome of this phage regulated the synthesis of a cytoplasmic element which might be either an enzyme geared to destroy a component formed by non-lysogenic bacteria which is specifically necessary for the vegetative development of phage $\lambda$, or a repressor which specifically inhibits one or more early reactions necessary for the multiplication of phages possessing the region $\mathrm{C} 1$ of $\lambda$. The fate of a bacterium infected by $\lambda$ would depend on the respective speed of the two processes, the synthesis of the repressor allowing of lysogenization on the one hand, and reactions which determine the triggering off of the vegetative phase on the other hand. They instance the action of chloramphenicol, which blocks protein synthesis and increases the probability of lysogenization, and moot the possibility of the repressor synthesized by the phage genome having a similar action. Lwoff (1961), discussing the immunity of a lysogenic bacterium to a mutant homologous phage, attributes this immunity to a specific repressor in the cytoplasm of the lysogenic bacterium which blocks specifically the initiation of the vegetative phase; here the question of prophage change does not arise, or at least is not obvious. Neither of these theories covers the interaction of lysogenic bacterium and superinfecting heterologous phage. If the curtailment of protein synthesis does in fact direct the superinfecting phage towards lysogenization and away from vegetative development, this cannot be the operative factor where both lysis and lysogenesis are suppressed. Our findings show that in many cases the repressor, whatever it may be, has this double action, and therefore appears to operate against some fundamental property common to all invading phages, whether destined to lysogenize or lyse. In certain cases, however, the repressor was more active against lysis, in others against lysogenesis. This, together with the irregularity and unpredictability of the reaction (well demonstrated by the reaction of Q1 (A4) superinfected with A2e, Q1 (A2c) superinfected with A2b and A2d, and present though less obvious in some other instances) suggests that each lysogenic strain may possess a repressor or repressors with specific characters rather than that all produce, with quantitative variations, a common type of repressor. It might be argued that as the end-result depends on the interaction of repressor and invading phage, the anomalous results could stem solely from an unusual degree of resistance or virulence on the part of the phage. Such a theory, which implies the presence in each lysogenic strain of varying concentrations of a common repressor as well as varying degrees of aggressiveness in the different phages, fails to explain why, for example, $\mathrm{A} 1 c$ is active against Q1 (A 2 $a$ ) and is almost completely suppressed by Q1 (A 2c), whereas A2b is well suppressed by Q1 (A2a) and active against Q1 (A 2c). While, therefore, different phages clearly have different degrees of aggressiveness, it is equally certain that the different lysogenic strains contain repressors of varying degrees of specificity.

The outstanding feature of these findings is the complexity of the picture which they reveal. As the host bacterium is common to all the lysogenic strains, the 
differing reactions must depend on variations in the phage component of the phagebacterium complex, and this despite the fact that certain basic characters of these phages show them to be closely related members of a well-defined group. Prolonged study has failed to reveal a formula which would enable any given set of reactions to be predicted with certainty: they appear to be in a category inviolate to statistical calculations and mathematical equations.

\section{The possible significance of the bacteriophage model}

In man and other animals the immunity conferred either by an attack of a virus disease or by the administration of an attenuated but living virus vaccine, and also certain associated phenomena of viral interference, raise problems of great interest. Animal viruses undoubtedly differ in many fundamental respects from bacterial viruses, just as metazoan cells differ from bacteria. Nevertheless, certain points of resemblance can be seen which suggest that a study of the well-established bacteriophage model might throw light on some of the problems of virus infection in man. As the immunity of the lysogenic bacterium is to all intents and purposes permanent, its most probable counterpart in human virus infection is the secure and usually life-long immunity resulting from attacks of certain diseases such as smallpox, yellow fever, measles and poliomyelitis. The solid immunity to further attacks of smallpox enjoyed by a person who has contracted and recovered from this disease is well known. That this immunity may be cellular and not humoral is suggested by the results of vaccination in infants suffering from hereditary hypo$\gamma$ globulinaemia in whom the infection may run a normal course (MacCallum, 1959), and also from the experiments of Friedman \& Baron (1961) who found that irradiated guinea-pigs, in which no detectable neutralizing antibody could be demonstrated, recovered from vaccinia infection as rapidly as non-irradiated animals, suggesting that production of neutralizing antibody was not necessary for recovery. Long and extensive experience leaves no doubt that a non-fatal attack of yellow fever confers life-long immunity to further infection with the virus. Sawyer (1931) found that the serum of 45 out of 60 subjects who had suffered from yellow fever 30 to 78 years previously-including 5 out of 6 with a 75 years' interval-protected susceptible monkeys from a challenge dose of yellow fever virus. Many of the 45 had no exposure to infection subsequent to the original illness. It is more than unlikely that the antibodies which protected the monkeys had been elaborated during the original attack and had persisted in the circulation for as long as 75 years: their presence clearly indicates some continuing and comparatively recent stimulation of the antibody-producing mechanism, and a possibility which suggests itself is that the susceptible cells (probably liver epithelium) which survived the original infection did so because they were 'lysogenized' by the virus; that this property, incorporated in the infected cells, was passed on to their progeny as a hereditary character; and that the virus which stimulated the antibody-producing mechanism was liberated from one of these lysogenized cells in which, as happens in bacterial cultures, the hypothetical 'provirus' had undergone vegetative development. If this is the explanation, the circulating antibodies may be incidentalthey are not to be detected in many who have suffered from yellow fever and so are undoubtedly immune--and the immunity which all recovered cases enjoy may rest fundamentally in the resistant lysogenized cells which in the non-immune 
subject would be open to attack by the virus. At least 3 types of poliovirus of different antigenic structure can cause paralytic poliomyelitis, and the accepted practice is to immunize with a vaccine composed of all three. Yet there is a record of an outbreak due to Type I virus being apparently cut short by mass immunization of the community with Type II attenuated virus vaccine (Hale et al. 1959). Could this result from a process, analogous to lysogenization of the susceptible cells, giving a cross-immunity similar to that shown by some of the lysogenic Q1 strains? These ideas are speculative, yet the examples given could be amplified and expanded in many ways and still remain so good a fit to the underlying theory that it is speculation which merits careful consideration.

\section{REFERENCES}

BertanI, G. (1953). Infections bactériophagiques secondaires des bactéries lysogènes. Ann. Inst. Pasteur, 84, 273.

Bertani, G. (1954). Studies on lysogenesis. III. Superinfection of lysogenic Shigella dysenteriae with temperate mutants of the carrier phage. J. Bact. 67, 696 .

Bertani, G. \& Six, E. (1958). Inheritance of prophage P2 in bacterial crosses. Virology, 6, 357.

Boyd, J. S. K. (1950). The symbiotic bacteriophages of Salmonella typhimurium. J. Path. Bact. 62, 501.

Boyd, J. S. K. (1951). Observations on the relationship of symbiotic and lytic bacteriophage. J. Path. Bact. 63, 445.

BoyD, J. S. K. (1954). Bacteriophage and heredity. Nature, Lond. 173, 1050.

BoYD, J. S. K. (1956). Immunity of lysogenic bacteria. Nature, Lond. 178, 141.

Boyd, J. S. K. \& Bmweld, D. E. (1957). The type A phages of Salmonella typhimurium: identification by a standardized cross-immunity test. J. gen. Microbiol. 16, 217.

Boyd, J. S. K. \& Bidwell, D. E. (1959). The Q1 (A) strains of Salmonella typhimurium: induction phenomena. J. gen. Microbiol. 21, 635.

Boyd, J. S. K. \& Bidwel, D. E. (1961). The type A phages of Salmonella typhimurium: observations on temperate phage and lysogenesis. J. gen. Microbiol. 26, 443.

Corten, D. (1959). A variant of phage $\mathbf{P 2}$ originating in Escherichia coli, Strain $\mathbf{B}^{{ }^{\prime}}$. Virology, 7, 112.

Friedman, R. M. \& Baron, S. (1961). The role of antibody in recovery from infection with vaccinia virus. J. Immunol. 87, 379.

GorRILl, R. H. (1957). Studies in lysogeny in staphylococci. J. gen. Microbiol. 17, 254.

Groman, N. B. (1955). Evidence for the active role of bacteriophage in the conversion of nontoxigenic Corynebacterium diphtheriae to toxin production. J. Bact. 69, 9.

Groman, N. B. \& Eaton, M. (1955). Genetic factors in Corynebacterium diphtheriae conversion. J. Bact. 70, 637.

Hale, J. H., Doraisingham, M., Kanagaratnam, K., Leong, K. W. \& Monteiro, E. S. (1959). Large-scale use of Sabin Type 2 attenuated poliovirus vaccine in Singapore during a Type 1 poliomyelitis epidemic. Brit. med. J. i, 1541.

$\mathrm{J}_{\mathrm{ACOB}}$, F. \& CAMPBELL, A. (1959). Sur le système de répression assurant l'immunité chez les bactéries lysogène. 'C.R. Acad. Sci., Paris, 248, 3219.

Levine, M. (1957). Mutations in the temperate phage P22 and lysogeny in Salmonella. Virology, 3, 22.

Lwoff, A. (1961). The dynamics of viral infections. Proc. Roy. Soc. B, 154, 1.

MacCallum, F. O. (1959). Some aspects of immunity in virus diseases. Proc. roy. Soc. Med. $52,135$.

Sawyer, W. A. (1931). The persistence of yellow fever immunity. J. prev. Med. 5, 413.

Six, E. (1960). Prophage substitution and curing in lysogenic cells superinfected with hetero-immune phage. J. Bact. 80, 728.

SIx, E. (1961). Inheritance of prophage P2 in superinfection experiments. Virology, 14, 220. ZiNDER, N. D.(1958). Lysogeny and superinfection immunityin Salmonella. Virology, 5, 291. 